\title{
ACM VECTOR BUNDLES ON PROJECTIVE SURFACES OF NONNEGATIVE KODAIRA DIMENSION
}

\author{
E. BALLICO, S. HUH AND J. PONS-LLOPIS
}

\begin{abstract}
Aвstract. In this paper we contribute to the construction of families of arithmetically Cohen-Macaulay $(\mathrm{aCM})$ indecomposable vector bundles on a wide range of polarized surfaces $\left(X, \mathscr{O}_{X}(1)\right)$ for $\mathscr{O}_{X}(1)$ an ample line bundle. In many cases, we show that for every positive integer $r$ there exists a family of indecomposable aCM vector bundles of rank $r$, depending roughly on $r$ parameters, and in particular they are of wild representation type. We also introduce a general setting to study the complexity of a polarized variety $\left(X, \mathscr{O}_{X}(1)\right)$ with respect to its category of aCM vector bundles. In many cases we construct indecomposable vector bundles on $X$ which are aCM for all ample line bundles on $X$.
\end{abstract}

\section{INTRODUCTION}

In many areas of mathematics it plays a central role to understand the complexity of the objects one is interested in. This complexity can be measured in many different ways. For instance, in representation theory of quivers, Gabriel's theorem states that a connected quiver supports only finitely many irreducible representations, i.e., of indecomposable modules over the associated path algebra, if and only if it is of type $A, D, E$. The classification of tame quivers as Euclidean graphs, or extended Dynkin diagrams, of type $\tilde{A}, \tilde{D}, \tilde{E}$ was obtained right after. Remarkably, any other quiver supports arbitrarily large families of indecomposable representations, i.e., they turn out to be of wild representation type.

Motivated by the results, similar questions were raised to understand the category of Cohen-Macaulay modules over an arbitrary k-algebra $R$. When $R:=\mathbf{k}\left[x_{0}, \ldots, x_{n}\right] / I$ is a graded algebra finitely generated in degree one over a field $\mathbf{k}$, Cohen-Macaulay modules correspond naturally to arithmetically CohenMacaulay sheaves over the closed subscheme $\operatorname{Proj}(R) \subset \mathbb{P}^{n}$; see [18].

Definition 1.1. A coherent sheaf $\mathscr{E}$ on a projective scheme $\left(X, \mathscr{O}_{X}(1)\right)$ is called arithmetically CohenMacaulay (for short, aCM) if the following conditions hold:

(i) $\mathscr{E}$ is locally Cohen-Macaulay, i.e. the stalk $\mathscr{E}_{x}$ has depth equal to $\operatorname{dim} \mathscr{O}_{X, x}$ for any point $x$ on $X$;

(ii) $H^{i}(\mathscr{E}(t))=0$ for all $t \in \mathbb{Z}$ and $i=1, \ldots, \operatorname{dim} X-1$.

The forementioned correspondence allowed to use a geometrical approach to this kind of questions. A milestone on this area was due to Horrocks, stating that the only indecomposable aCM sheaf on $\mathbb{P}^{n}$, up to twist, is $\mathscr{O}_{\mathbb{P} n}$; see [15]. A similar classification was obtained for a smooth quadric hypersurface $Q \subset \mathbb{P}^{n}$ : there exist, besides the structural sheaf $\mathscr{O}_{Q}$, only one (for $n$ even) or two (for $n$ odd) irreducible aCM sheaves, the well-studied Spinor bundles; see [19]. The combined work of many mathematicians allowed to complete the list of projective schemes -of positive dimension- supporting a finite number of aCM sheaves, called the varieties of finite aCM-representation type: they are either a projective space $\mathbb{P}^{n}$, a smooth quadric hypersurface $X \subset \mathbb{P}^{n}$, a cubic scroll in $\mathbb{P}^{4}$, the Veronese surface in $\mathbb{P}^{5}$ or a rational normal curve; see [8].

2010 Mathematics Subject Classification. Primary: 14F05; Secondary: 13C14, 16 G60.

Key words and phrases. polarized surfaces, arithmetically Cohen-Macaulay sheaf, wild representation type.

The first author is partially supported by GNSAGA of INDAM (Italy) and MIUR PRIN 2015 'Geometria delle varietà algebriche'. The second author is supported by the National Research Foundation of Korea(NRF) grant funded by the Korea government(MSIT) (No. 2018R1C1A6004285 and No. 2016R1A5A1008055). The third author is supported by a Postdoctoral Fellowship DISIM 2017-B0010. 
The next degree of complexity is offered by the elliptic curves: in this case, vector bundles of a given rank and degree on an elliptic curve $C$ are in bijection with the points of $C$; see [1]. They are called varieties of tame aCM-representation type. In [9] it was shown that smooth quartic surface scrolls in $\mathbb{P}^{5}$ are also tame. Notice that all the projective schemes $X \subset \mathbb{P}^{n}$ mentioned until now are arithmetically Cohen-Macaulay, namely the coordinate ring $R:=\mathbf{k}\left[x_{0}, \ldots, x_{n}\right] / I_{X}$ is a Cohen-Macaulay ring. Indeed, the represention type of the remaining aCM projective schemes $X \subset \mathbb{P}^{n}$ was set in [10]: they support arbitrarily large families of indecomposable non-isomorphic aCM sheaves. They are, therefore, of wild aCM-representation type.

On the other hand, up to our knowledge, a broader problem has been much less studied: which are the possible dimensions of families of aCM irreducible sheaves on polarized schemes $\left(X, \mathscr{O}_{X}(1)\right)$, where the only requirement for the line bundle $\mathscr{O}_{X}(1)$ is to be ample. With this setting it is proved in [6] and [7] that polarized surfaces $\left(S, \mathscr{O}_{S}(1)\right)$ such that $p_{g}=0, q=0$ or 1 , and $\mathscr{O}_{S}(1)$ is very ample with $h^{1}\left(\mathscr{O}_{S}(1)\right)=0$ are of wild representation type. Indeed, the aCM vector bundles witnessing wilderness own a special property: they have the maximal permitted number of global sections, namely they are the so-called $U l$ rich vector bundles. Again for $\mathscr{O}_{X}(1)$ very ample, it is proved in [22] that for polarized varieties $\left(X, \mathscr{O}_{X}(1)\right)$ of dimension at least two, the embedding given by $\mathscr{O}_{X}(l)$ with $l \geq 3$ is of wild representation type under some mild assumptions on $\mathscr{O}_{X}(1)$.

The goal of the present paper is to contribute to this set of problems: we are constructing families of aCM vector bundles on a large range of polarized integral surfaces $\left(X, \mathscr{O}_{X}(1)\right)$. In the following Theorem we summarize the results obtained:

Theorem 1.2. Let $X$ be an integral projective surface with a fixed ample line bundle $\mathscr{O}_{X}(1)$ listed below. Then for each integer $r \geq 2$ there exists an $b_{X}(r)$-dimensional irreducible family $\left\{\mathscr{E}_{\alpha}\right\}_{\alpha \in \Gamma}$ of indecomposable aCM vector bundles of rank $r$ on $X$ such that for each $\alpha \in \Gamma$ there are only finitely many $\beta \in \Gamma$ with $\mathscr{E}_{\alpha} \cong \mathscr{E}_{\beta}$.

\begin{tabular}{|c|c|c|}
\hline no. & $X$ & $b_{X}(r)$ \\
\hline 1 & $\begin{array}{c}\pi: X \rightarrow Y \text { a birational morphism with } \omega_{Y} \cong \mathscr{O}_{Y} \text { and } q(Y)=0 \\
\text { such that } \pi^{-1}\left(Y_{\text {sing }}\right) \cong Y_{\text {sing }}\end{array}$ & $2 r$ \\
\hline 2 & $\omega_{X} ¥ \mathscr{O}_{X}$ locally free with $h^{0}\left(\omega_{X}\right)=0$ and $h^{0}\left(\omega_{X}^{\otimes 2}\right)=1$, and $q(X)=0$ & $2\left\lceil\frac{r}{2}\right\rceil$ \\
\hline 3 & smooth and $q(X)=1$ with $\omega_{X}^{\vee} \otimes \mathscr{O}_{X}(1)$ trivial or ample & 1 \\
\hline 4 & $\begin{array}{l}\pi: X \rightarrow Y \text { a birational morphism with an abelian surface } Y \\
\qquad \text { and } \omega_{X}^{\vee} \otimes \mathscr{O}_{X}(1) \text { trivial or ample }\end{array}$ & $r+1$ \\
\hline 5 & $\pi: X \rightarrow Y$ a birational morphism with a hyperelliptic surface $Y$ & 1 \\
\hline 6 & $\omega_{X} \cong \mathscr{O}_{X}(1)$ with $h^{1}\left(\omega_{X}^{\otimes n}\right)=0$ for all $n \in \mathbb{Z}$ and $p_{g} \geq 3$ & $r$ \\
\hline
\end{tabular}

Theorem 1.2 shows that the projective surfaces of Kodaira dimension zero, possibly with singularity, are of wild representation type, except the case of hyperelliptic surfaces. G. Casnati proved in [7] that hyperelliptic surfaces are of wild representation type with respect to a very ample polarization. Note that we do not assume in Theorem 1.2 that $X$ is minimal or $\mathscr{O}_{X}(1)$ is very ample, while the result in [7] is more powerful in a sense that it gives wildness with respect to Ulrich vector bundles.

The strategy for Theorem 1.2 is two-fold. One is to consider zero-dimensional subschemes of length equal to the second Chern class of the aCM vector bundles in consideration, from which we construct aCM vector bundles of arbitrary rank by a series of extensions. The cases no. 1,2 and 6 are handled by this method respectively in Theorem 2.4, Theorem 3.5 and Theorem 5.4 in case no. 6, for the construction of a family of aCM vector bundles of rank $r$ even, it is enough to suppose that $p_{g} \geq 2$. The second strategy is to consider a family of aCM line bundles, parametrized by a non-empty open Zariski subset of $\operatorname{Pic}^{0}(X)$, from which we construct aCM vector bundles of arbitrary rank by iterated extensions. the cases no. 3, 4 and 5 are handled by this method respectively in Proposition 4.1, Theorem 1.3 and Proposition 4.5 , 
Based on the results in Theorem 1.2 we introduce a set-up to measure the complexity of a polarized variety $\left(X, \mathscr{O}_{X}(1)\right)$. Define

$$
a_{X, \mathscr{O}_{X}(1)}(r):=\sup _{\Gamma}\left\{\begin{array}{l|c}
\operatorname{dim} \Gamma & \begin{array}{c}
\Gamma \text { runs over the parameter spaces of indecomposable } \\
\text { aCM vector bundles of rank } r \text { on } X
\end{array}
\end{array}\right\}
$$

with the convention that $a_{X, \mathscr{O}_{X}(1)}(r)=-\infty$ if there is no indecomposable aCM vector bundle of rank $r$. Then we have $a_{X, \mathscr{O}_{X}(1)}(r) \geq b_{X}(r)$ for the surfaces listed in Theorem1.2. We also define

$$
a_{X}(r):=\sup \left\{a_{X, \mathscr{O}_{X}(1)}(r) \mid \mathscr{O}_{X}(1) \text { ample }\right\}, a_{X}^{\prime}(r):=\inf \left\{a_{X, \mathscr{O}_{X}(1)}(r) \mid \mathscr{O}_{X}(1) \text { ample }\right\} .
$$

In many construction of aCM vector bundles, the polarization is assumed to be very ample, in which case we give similar definitions for $a_{X}(r)$ and $a_{X}^{\prime}(r)$, if we consider only very ample polarizations in their definitions. Then we may raise several questions.

- For a given $X$, what can be said about the following limits?

$$
\limsup _{r \rightarrow \infty} a_{X}(r), \limsup _{r \rightarrow \infty} a_{X}^{\prime}(r), \liminf _{r \rightarrow \infty} a_{X}(r) \text { and } \liminf _{r \rightarrow \infty} a_{X}^{\prime}(r)
$$

- What can be said about following suprema

$$
\sup _{X}\left\{a_{X}(r)\right\} \text { and } \sup _{X}\left\{a_{X}^{\prime}(r)\right\},
$$

where $X$ runs over all smooth projective varieties, all varieties with a prescribed Kodaira dimension or all varieties in a prescribed interesting class, e.g. K3 surfaces?

In those questions with $\left(X, \mathscr{O}_{X}(1)\right.$ polarized surfaces, we may allow singular surfaces, but locally CM, e.g. normal or singularity with embedded dimension at most three, so that we may consider non-locally free aCM sheaves. We do not know if we may obtain bigger dimensional families of indecomposable aCM sheaves by considering non-locally free aCM sheaves.

For higher dimensional smooth varieties we prove the following result.

Theorem 1.3. Let $X$ be a smooth projective variety of dimension $n \geq 2$, birational to an abelian variety and fix an ample line bundle $\mathscr{O}_{X}(1)$ with $\omega_{X}^{\vee} \otimes \mathscr{O}_{X}(1)$ ample. Then $X$ is wild with respect to $\mathscr{O}_{X}(1)$ and

$$
a_{X, \mathscr{O}_{X}(1)}(r) \geq(n-1) r+1 .
$$

For the proof of Theorem 1.3 we use in an essential way a construction by S. Mukai of vector bundles on abelian varieties in [21], a generic vanishing for smooth varieties with maximal Albanese dimension in [12, 13] and results on the local Hilbert schemes in [5, 11].

Remark 1.4. In cases no. 1, 2 and 6 of Theorem 1.2 the indecomposable vector bundles that we construct are aCM for any ample line bundles on $X$. On the other hand, in cases no. 3, 4 and 5 of Theorem 1.2 and Theorem 1.3 the indecomposable vector bundles that we construct are aCM for every ample line bundles $\mathscr{O}_{X}(1)$ with $\omega_{X}^{\vee} \otimes \mathscr{O}_{X}(1)$ ample.

Recall from Theorem 1.2 that we obtain irreducible families of indecomposable aCM vector bundles of rank $r$ on several projective surfaces, whose dimensions are at most linear polynomials in $r$. Nonetheless, we may not expect that $a_{X, \mathscr{O}_{X}(1)}(r)$ is linear in $r$ for any projective surface. Indeed, Remark1.5]shows that for $X$ as in Theorem 1.5 with $n \geq 3$ we get a lower bound for $a_{X, \mathscr{O}_{X}(1)}(r)$ greater than linear, but less than quadratic, in $r$.

Remark 1.5. Let $X$ be as in Theorem 1.3 . For $n \geq 3$ and $r \gg 0, \mathbb{U}_{r}$ is reducible and there are positive constants $\alpha_{n}$ and $\beta_{n}$ such that

$$
\alpha_{n} r^{2-2 / n} \leq \operatorname{dim} B_{f}[r] \leq \beta_{n} r^{2-2 / n}
$$

by [5] and [11, page 6]. Then from $\operatorname{dim} B_{f}[r] \leq a_{X, \mathscr{O}_{X}(1)}(r)$ we get

$$
\liminf _{r \rightarrow \infty} \frac{a_{r}\left(X, \mathscr{O}_{X}(1)\right)}{r^{2-2 / n}}>0 .
$$


On the other hand, in Section 6 we suggest examples of smooth surfaces of general type with at least a quadratic lower bound for $a_{X, \mathscr{O}_{X}(1)}(r)$.

We would like thank C. Ciliberto for suggesting this problem.

\section{K3-LIKE SURFACES}

In this section we assume that $X$ is integral with $\omega_{X} \cong \mathscr{O}_{X}$ and $q(X)=0$. Set $\tilde{g}:=h^{0}\left(\mathscr{O}_{X}(1)\right)$; if $X$ is a K3 surface, then we have $2 \tilde{g}-4=d$ and $g:=\tilde{g}-1$ is called the genus of $X$.

Proposition 2.1. For each $r \in \mathbb{Z}$ with $2 \leq r \leq \tilde{g}$, there exists an indecomposable aCM vector bundle $\mathscr{E}$ of rank $r$ on $X$ with $\operatorname{det}(\mathscr{E}) \cong \mathscr{O}_{X}$ and $c_{2}(\mathscr{E})=r$

Proof. Take a general set of points $S \subset X_{\text {reg with }}|S|=r$ and let $\mathscr{E}$ be a general sheaf fitting into the following exact sequence

$$
0 \rightarrow \mathscr{O}_{X}^{\oplus(r-1)} \stackrel{j}{\rightarrow} \mathscr{E} \rightarrow \mathscr{I}_{S, X} \rightarrow 0 .
$$

Note that $\operatorname{ext}_{X}^{1}\left(\mathscr{I}_{S, X}, \mathscr{O}_{X}\right)=h^{1}\left(\mathscr{I}_{S, X}\right)=r-1$ and the sheaf $\operatorname{Im}(j)$ is the image of the evaluation map $H^{0}(\mathscr{E}) \otimes \mathscr{O}_{X} \rightarrow \mathscr{E}$. By generality of the extension (1) we may choose a basis $\left\{\varepsilon_{1}, \ldots, \varepsilon_{r-1}\right\}$ of $\operatorname{Ext}_{X}^{1}\left(\mathscr{I}_{S, X}, \mathscr{O}_{X}\right)$ inducing (1). In particular, $\mathscr{E}$ has no trivial factor. In case $r=2$, the sheaf $\mathscr{E}$ is locally free from the CayleyBacharach condition. For $r>2$, the vector bundle $\mathscr{F} \oplus \mathscr{O}_{X}^{\oplus(r-2)}$ with a vector bundle $\mathscr{F}$ of rank two fitting into (1) as the middle term for $r=2$, also fits into (1) as the middle term. Since local freeness is an open condition, the sheaf $\mathscr{E}$ is locally free.

Assume $\mathscr{E} \cong \mathscr{F}_{1} \oplus \mathscr{F}_{2}$ with $\operatorname{rank}\left(\mathscr{F}_{1}\right)=s$ and $0<s<r$. For each $i \in\{1,2\}$, let $\mathscr{G}_{i} \subseteq \mathscr{F}_{i}$ be the image of the evaluation map $H^{0}\left(\mathscr{F}_{i}\right) \otimes \mathscr{O}_{X} \rightarrow \mathscr{F}_{i}$ with $s_{i}:=\operatorname{rank}\left(\mathscr{G}_{i}\right)$. Then we get $\mathscr{G}_{1} \oplus \mathscr{G}_{2} \cong \mathscr{O}_{X}^{\oplus(r-1)}$. In particular, each $\mathscr{G}_{i}$ is trivial and $s_{1} \in\{s, s-1\}$. Note that $\left(\mathscr{F}_{1} / \mathscr{G}_{1}\right) \oplus\left(\mathscr{F}_{2} / \mathscr{G}_{2}\right) \cong \mathscr{I}_{S, X}$ has no torsion. If $s_{1}=s$, then we get $\mathscr{F}_{1} / \mathscr{G}_{1} \cong 0$, i.e. $\mathscr{F}_{1} \cong \mathscr{O}_{X}^{\oplus S}$, which is impossible since $\mathscr{E}$ has no trivial factor. If $s_{1}=s-1$, then we would get a contradiction similarly from $\mathscr{F}_{2} \cong \mathscr{O}_{X}^{\oplus(r-s)}$. Thus $\mathscr{E}$ is indecomposable.

Then it remains to show that $\mathscr{E}$ is aCM. Since $h^{0}\left(\mathscr{O}_{S}\right) \leq h^{0}\left(\mathscr{O}_{X}(1)\right)$ and $S$ is general, we have $h^{1}\left(\mathscr{I}_{S, X}(t)\right)=$ 0 for all $t>0$. Now $\left\{\varepsilon_{1}, \ldots, \varepsilon_{r-1}\right\}$ is a basis for $\operatorname{Ext}_{X}^{1}\left(\mathscr{I}_{S, X}, \mathscr{O}_{X}\right)$ and so it induces an isomorphism $H^{1}\left(\mathscr{I}_{S, X}\right) \rightarrow$ $H^{2}\left(\mathscr{O}_{X}^{\oplus(r-1)}\right)$. Thus we have $h^{0}(\mathscr{E}(t))=0$ for all $t \geq 0$. For any $\lambda \in \mathbf{k}$, let $\mathscr{E}_{\lambda}$ denote the middle term of the extension corresponding to $\left(\varepsilon_{1}, \lambda \varepsilon_{2}, \ldots, \lambda \varepsilon_{r-1}\right)$; we have $\mathscr{E}_{\lambda} \cong \mathscr{E}$ for $\lambda \neq 0$ and $\mathscr{E}_{0} \cong \mathscr{G} \oplus \mathscr{O}_{X}^{\oplus(r-2)}$ with $\mathscr{G}$ induced by the extension $\varepsilon_{1}$. As above we see that $h^{1}(\mathscr{G}(t))=0$ for all $t \geq 0$. Since $\mathscr{G}$ is locally free from the Cayley-Bacharach condition and generality of $\varepsilon_{1}$, we use Serre's duality to obtain $h^{1}(\mathscr{G}(t))=h^{1}(\mathscr{G}(-t))=0$ for $t<0$. Thus $\mathscr{E}_{0}$ is aCM. Now using the semicontinuity theorem for cohomology, we obtain $h^{1}(\mathscr{E}(t))=0$ because $\mathscr{E}_{\lambda} \cong \mathscr{E}$.

Remark 2.2. Consider the exact sequence (1) with $r=2$. Since $\operatorname{ext}_{X}^{1}\left(\mathscr{I}_{S, X}, \mathscr{O}_{X}\right)=h^{1}\left(\mathscr{I}_{S, X}\right)=1$, there exists a unique nontrivial extension of $\mathscr{I}_{S, X}$ by $\mathscr{O}_{X}$; denote its middle term by $\mathscr{G}_{S}$. Since the Cayley-Bacharach condition is satisfied, the sheaf $\mathscr{G}_{S}$ is an aCM vector bundle of rank two on $X$.

Theorem 2.3. For each integer $2 \leq r \leq \tilde{g}$, there exists a $2 r$-dimensional family $\left\{\mathscr{E}_{\alpha}\right\}_{\alpha \in \Gamma}$ of indecomposable aCM vector bundles of rank $r$ on $X$ with $\operatorname{det}\left(\mathscr{E}_{\alpha}\right) \cong \mathscr{O}_{X}$ and $c_{2}\left(\mathscr{E}_{\alpha}\right)=r$ such that for each $\alpha \in \Gamma$ there are only finitely many $\beta \in \Gamma$ with $\mathscr{E}_{\beta} \cong \mathscr{E}_{\alpha}$.

Proof. For any subset $S \subset X_{\text {reg }}$ with $|S|=r$, define $\mathbb{E}^{\prime}(S)$ to be the subset of $\mathbb{E}(S):=\operatorname{Ext}_{X}^{1}\left(\mathscr{I}_{S, X}, \mathscr{O}_{X}^{\oplus(r-1)}\right)$, consisting of all extensions whose corresponding middle terms are aCM and indecomposable vector bundles. By Proposition 2.1 $\mathbb{E}^{\prime}(S)$ is a non-empty open subset of $\mathbb{E}(S)$ and each $[\mathscr{E}] \in \mathbb{E}^{\prime}(S)$ has trivial determinant with $c_{2}(\mathscr{E})=r$.

Letting $\mathbb{U}:=\left\{S \subset X_{\text {reg }}|| S \mid=r\right\}$, there is a vector bundle $\mathcal{V}$ of rank $(r-1)^{2}$ on $\mathbb{U}$ with $\mathbb{E}(S)$ as its fibre over $S \in \mathbb{U}$, since $\operatorname{ext}_{X}^{1}\left(\mathscr{I}_{S, X}, \mathscr{O}_{X}^{\oplus(r-1)}\right)=(r-1)^{2}$ for all $S \in \mathbb{U}$. Then there is a non-empty open subset $\mathscr{V}^{\prime} \subset \mathcal{V}$ with $V_{\mid S}^{\prime}=\mathbb{E}^{\prime}(S)$ for a general $S \in \mathbb{U}$. Thus there exists an irreducible variety $\Gamma \subset V^{\prime}$ such that the restriction of the map $\mathcal{V} \rightarrow \mathbb{U}$ to $\Gamma$ is quasi-finite and dominant. In particular, we have $\operatorname{dim} \Gamma=\operatorname{dim} \mathbb{U}=2 r$. 
For $[\mathscr{E}] \in \mathbb{E}^{\prime}(S)$ we have $h^{0}(\mathscr{E})=r-1$ and the cokernel of the evaluation map $H^{0}(\mathscr{E}) \otimes \mathscr{O}_{X} \rightarrow \mathscr{E}$ is isomorphic to $\mathscr{I}_{S, X}$. Thus for $[\mathscr{E}] \in \mathbb{E}^{\prime}(S)$ and $[\mathscr{F}] \in \mathbb{E}^{\prime}\left(S^{\prime}\right)$ with $S \neq S^{\prime} \in \mathbb{U}$, we have $\mathscr{E} \not \mathscr{E}^{\prime}$. Since the map $\Gamma \rightarrow \mathbb{U}$ is quasi-finite, the variety $\Gamma$ satisfies the requirements for the assertion.

Theorem 2.4. For each integer $r \geq 2$, there exists an $2 r$-dimensional family $\left\{\mathscr{E}_{\alpha}\right\}_{\alpha \in \Gamma}$ of indecomposable aCM vector bundles of rank $r$ on $X$ with $\operatorname{det}\left(\mathscr{E}_{\alpha}\right) \cong \mathscr{O}_{X}$ and $c_{2}\left(\mathscr{E}_{\alpha}\right)=r$ such that for each $\alpha \in \Gamma$ there are only finitely many $\beta \in \Gamma$ with $\mathscr{E}_{\beta} \cong \mathscr{E}_{\alpha}$.

For the proof of Theorem 2.4 we collect numerous technical results below. We fix subsets $S_{0}, \ldots, S_{m} \subset$ $X_{\text {reg with }}\left|S_{0}\right|=3$ and $\left|S_{i}\right|=2$ for all $1 \leq i \leq m$ such that $S_{i} \cap S_{j}=\varnothing$ for any $i \neq j$.

Set $\square\left(S_{1}\right):=\left\{\mathscr{I}_{S_{1}, X}\right\}$ and define $\square\left(S_{1}, \ldots, S_{i}\right)$ for $i \geq 2$ inductively to be the set of all sheaves admitting an extension of $\mathscr{I}_{S_{i}, X}$ by an element in $\llbracket\left(S_{1}, \ldots, S_{i-1}\right)$. Thus for each $i \geq 2$ each sheaf $\mathscr{J} \in \llbracket\left(S_{1}, \ldots, S_{i}\right)$ admits the following exact sequence for some $\mathscr{J}^{\prime} \in \llbracket\left(S_{1}, \ldots, S_{i-1}\right)$

$$
0 \rightarrow \mathscr{J}^{\prime} \rightarrow \mathscr{J} \rightarrow \mathscr{I}_{S_{i}, X} \rightarrow 0 .
$$

For a subset $N=\left\{i_{1}, \ldots, i_{k}\right\} \subset\{1, \ldots, i\}$ with $i_{1}<\ldots<i_{k}$, we denote $\square\left(S_{i_{1}}, \ldots, S_{i_{k}}\right)$ by $\square\left(S_{j} ; j \in N\right)$.

Set $\mathbb{J}\left(\varnothing ; S_{0}\right):=\left\{\mathscr{I}_{S_{0}, X}\right\}$ and define $\mathbb{}\left(S_{1}, \ldots, S_{i} ; S_{0}\right)$ to be the set of all isomorphism classes of extensions of $\mathscr{I}_{S_{0}, X}$ by an element in $\llbracket\left(S_{1}, \ldots, S_{i}\right)$. Similarly we define $\mathbb{J}\left(S_{j} ; j \in N ; S_{0}\right)$.

Lemma 2.5. Each sheaf $\mathscr{J} \in \mathbb{\square}\left(S_{1}, \ldots, S_{i}\right)$ admits an exact sequence

$$
0 \rightarrow \mathscr{J} \stackrel{\iota}{\rightarrow} \mathscr{J}^{\vee \vee} \cong \mathscr{O}_{X}^{\oplus i} \rightarrow \mathscr{O}_{S_{1} \cup \cdots \cup S_{i}} \rightarrow 0,
$$

where the map $\mathrm{s}$ is the double dual map. In particular, we have $h^{0}(\mathscr{J})=0$ and $h^{1}(\mathscr{J})=h^{2}(\mathscr{J})=i$.

Proof. The assertion is clear for $i=1$, i.e. $\mathscr{J}=\mathscr{I}_{S_{1}, X}$. Assume $i \geq 2$ and consider an exact sequence (2) with $\mathscr{J}^{\prime} \in \llbracket\left(S_{1}, \ldots, S_{i-1}\right)$. By inductive hypothesis, the assertion holds for $\mathscr{J}^{\prime}$ and $\mathscr{I}_{S_{i}, X}$ and we get the following commutative diagram:

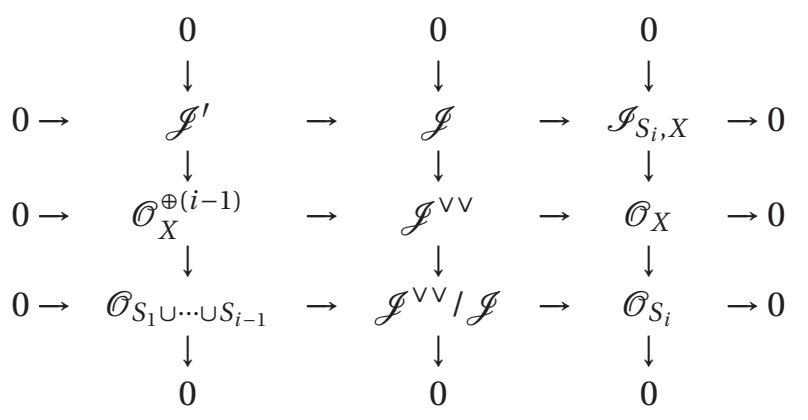

Since $\operatorname{ext}_{X}^{1}\left(\mathscr{O}_{X}, \mathscr{O}_{X}\right)=h^{1}\left(\mathscr{O}_{X}\right)=0$, we get $\mathscr{J}^{\vee \vee} \cong \mathscr{O}_{X}^{\oplus i}$ from the second horizontal sequence. From the third horizontal sequence, we get $\mathscr{J}^{\vee \vee} / \mathscr{J} \cong \mathscr{O}_{S_{1} \cup \cdots \cup S_{i}}$, because $S_{i}$ 's are disjoint to each other. Then we get the exact sequence (3). The vanishing $H^{0}(\mathscr{J})=0$ can be obtained by induction on $i$ and $h^{1}(\mathscr{J})=h^{2}(\mathscr{J})=i$ can be obtained from (3).

Remark 2.6. By the same argument in the proof of Lemma2.5, we have an exact sequence

$$
0 \rightarrow \tilde{\mathscr{J}} \rightarrow \tilde{\mathcal{J}}^{\vee \vee} \cong \mathscr{O}_{X}^{\oplus(i+1)} \rightarrow \mathscr{O}_{S_{0} \cup S_{1} \cup \cdots \cup S_{i}} \rightarrow 0,
$$

for $\tilde{\mathscr{J}} \in \mathbb{J}\left(S_{1}, \ldots, S_{i} ; S_{0}\right)$. This gives $h^{0}(\tilde{\mathscr{J}})=0, h^{1}(\tilde{\mathscr{J}})=i+2$ and $h^{2}(\tilde{\mathscr{J}})=i+1$.

Lemma 2.7. For a sheaf $\mathscr{J} \in \llbracket\left(S_{1}, \ldots, S_{i}\right)$ and any finite subset $A \subset X$,

(i) if $A \nsubseteq S_{j}$ for all $1 \leq j \leq i$, then we have $\operatorname{Hom}_{X}\left(\mathscr{J}, \mathscr{I}_{A, X}\right)=0$;

(ii) if $A \nsupseteq S_{j}$ for some $1 \leq j \leq i$, then we have $\operatorname{Hom}_{X}\left(\mathscr{I}_{A, X}, \mathscr{J}\right)=0$.

Proof. We only prove part (i), because part (ii) can be obtained similarly. Let us use induction on $i$; the case $i=1$ is true, because $A \nsubseteq S_{1}$ is equivalent to $\operatorname{Hom}_{X}\left(\mathscr{I}_{S_{1}, X}, \mathscr{I}_{A, X}\right)=0$. Now assume $i \geq 2$ and consider the sequence (2) with $\mathscr{J} \in \square\left(S_{1}, \ldots, S_{i-1}\right)$. Since $\operatorname{Hom}_{X}\left(\mathscr{I}_{S_{i}, X}, \mathscr{I}_{A, X}\right)=0$, any map $f \in \operatorname{Hom}_{X}\left(\mathscr{J}, \mathscr{I}_{A, X}\right)$ is 
uniquely determined by $f^{\prime} \in \operatorname{Hom}_{X}\left(\mathscr{J}^{\prime}, \mathscr{I}_{A, X}\right)$. The inductive assumption gives $f^{\prime}=0$ and so we have $f=0$.

Lemma 2.8. We have $\operatorname{ext}_{X}^{1}\left(\mathscr{I}_{S_{i+1}, X}, \mathscr{J}\right)=2 i$ for $\mathscr{J} \in \llbracket\left(S_{1}, \ldots, S_{i}\right)$.

Proof. Let $S:=S_{1} \cup \cdots \cup S_{i}$ and apply the functor $\operatorname{Hom}_{X}\left(\mathscr{I}_{S_{i+1}, X},-\right)$ to the sequence (3) to obtain

$$
\begin{aligned}
0 & \rightarrow \operatorname{Hom}_{X}\left(\mathscr{I}_{S_{i+1}, X}, \mathscr{J}\right) \rightarrow \operatorname{Hom}_{X}\left(\mathscr{I}_{S_{i+1}, X}, \mathscr{O}_{X}^{\oplus i}\right) \rightarrow \operatorname{Hom}_{X}\left(\mathscr{I}_{S_{i+1}, X}, \mathscr{O}_{S}\right) \\
& \rightarrow \operatorname{Ext}_{X}^{1}\left(\mathscr{I}_{S_{i+1}, X}, \mathscr{J}\right) \rightarrow \operatorname{Ext}_{X}^{1}\left(\mathscr{I}_{S_{i+1}, X}, \mathscr{O}_{X}^{\oplus i}\right) \rightarrow \operatorname{Ext}_{X}^{1}\left(\mathscr{I}_{S_{i+1}, X}, \mathscr{O}_{S}\right) .
\end{aligned}
$$

Here, we have $\operatorname{hom}_{X}\left(\mathscr{I}_{S_{i+1}, X}, \mathscr{O}_{X}^{\oplus i}\right)=i=\operatorname{ext}_{X}^{1}\left(\mathscr{I}_{S_{i+1}, X}, \mathscr{O}_{X}^{\oplus i}\right)$. We also get hom ${ }_{X}\left(\mathscr{I}_{S_{i+1}, X}, \mathscr{O}_{S}\right)=2 i$, because $S$ is disjoint from $S_{i+1}$. Now apply the functor $\operatorname{Hom}_{X}\left(-, \mathscr{O}_{S}\right)$ to the standard exact sequence for $S_{i+1} \subset X$ to obtain

$$
\operatorname{Ext}_{X}^{1}\left(\mathscr{O}_{X}, \mathscr{O}_{S}\right) \rightarrow \operatorname{Ext}_{X}^{1}\left(\mathscr{I}_{S_{i+1}, X}, \mathscr{O}_{S}\right) \rightarrow \operatorname{Ext}_{X}^{2}\left(\mathscr{O}_{S_{i+1}}, \mathscr{O}_{S}\right) .
$$

Here, we have $\operatorname{ext}_{X}^{1}\left(\mathscr{O}_{X}, \mathscr{O}_{S}\right)=h^{1}\left(\mathscr{O}_{S}\right)=0$ and $\operatorname{ext}_{X}^{2}\left(\mathscr{O}_{S_{i+1}}, \mathscr{O}_{S}\right)=0$. In particular, we get $\operatorname{ext}_{X}^{1}\left(\mathscr{I}_{S_{i+1}, X}, \mathscr{O}_{S}\right)=$ 0 . Finally, apply the functor $\operatorname{Hom}_{X}\left(\mathscr{I}_{S_{i+1}, X},-\right)$ to the sequence (2) to have

$$
\operatorname{Hom}_{X}\left(\mathscr{I}_{S_{i+1}, X}, \mathscr{J}^{\prime}\right) \rightarrow \operatorname{Hom}_{X}\left(\mathscr{I}_{S_{i+1}, X}, \mathscr{J}\right) \rightarrow \operatorname{Hom}_{X}\left(\mathscr{I}_{S_{i+1}, X}, \mathscr{I}_{S_{i}, X}\right) .
$$

Since $S_{i} \cap S_{i+1}=\varnothing$, we get $\operatorname{hom}_{X}\left(\mathscr{I}_{S_{i+1}, X}, \mathscr{I}_{S_{i}, X}\right)=0$. By inductive hypothesis, we get $\operatorname{hom}_{X}\left(\mathscr{I}_{S_{i+1}, X}, \mathscr{J}^{\prime}\right)=$ 0 . Thus we have $\operatorname{hom}_{X}\left(\mathscr{I}_{S_{i+1}, X}, \mathscr{J}\right)=0$ and we get the assertion.

Remark 2.9. Similarly as in the proof of Lemma2.8, we see that $\operatorname{ext}_{X}^{1}\left(\mathscr{I}_{S_{0}, X}, \mathscr{J}\right)=3 i$ for any $\mathscr{J} \in \mathbb{\square}\left(S_{1}, \ldots, S_{i}\right)$. In particular, there exists a non-trivial extension

$$
0 \rightarrow \mathscr{J} \rightarrow \tilde{\mathscr{J}} \rightarrow \mathscr{I}_{S_{0}, X} \rightarrow 0 .
$$

In this case, we have $\operatorname{ext}_{X}^{1}\left(\mathscr{I}_{S_{0}, X}, \mathscr{O}_{X}^{\oplus i}\right)=2 i$ and the other numeric data in the proof of Lemma2.8 are all same.

Lemma 2.10. For each $i \geq 1$, there exists an indecomposable sheaf $\mathscr{J} \in \llbracket\left(S_{1}, \ldots, S_{i}\right)$.

Proof. Since $\mathscr{I}_{S_{1}, X}$ has rank one and $X$ is an integral variety, $\mathscr{I}_{S_{1}, X}$ is indecomposable. Thus we may assume $i \geq 2$. Note that each $\mathscr{I}_{S_{j}, X}$ has the same Hilbert polynomial with respect to any polarization $\mathscr{O}_{X}(1)$. Thus any sheaf in $\llbracket\left(S_{1}, \ldots, S_{i}\right)$ is strictly semistable with $\oplus_{j=1}^{i} \mathscr{I}_{S_{j}, X}$ as its Jordan-Hölder grading. Let $\mathscr{J}$ be a general sheaf fitting into an exact sequence

$$
0 \rightarrow \oplus_{j=1}^{i-1} \mathscr{I}_{S_{j}, X} \stackrel{f}{\rightarrow} \mathscr{J} \stackrel{g}{\rightarrow} \mathscr{I}_{S_{i}, X} \rightarrow 0
$$

and assume that $\mathscr{J}$ is decomposable, say $\mathscr{J} \cong \mathscr{A}_{1} \oplus \cdots \oplus \mathscr{A}_{h}$ with $h \geq 2$ and each $\mathscr{A}_{j}$ indecomposable. Since $\mathscr{J}$ is strictly semistable with $g r(\mathscr{J}) \cong \oplus_{j=1}^{i} \mathscr{I}_{S_{j}, X}$, there is a subset $N_{j} \subset\{1, \ldots, i\}$ for each $j \in\{1, \ldots, h\}$ such that $\operatorname{gr}\left(\mathscr{A}_{j}\right) \cong \oplus_{k \in N_{j}} \mathscr{I}_{S_{k}, X}$. Note that $\left\{N_{j} \mid 1 \leq j \leq h\right\}$ forms a partition of $\{1, \ldots, i\}$ with each $N_{j}$ nonempty.

Assume first that $\left|N_{j}\right|=1$ for all $j$. Then we have $\mathscr{J} \cong \oplus_{j=1}^{i} \mathscr{I}_{S_{j}, X}$. Since we have $\operatorname{Hom}_{X}\left(\mathscr{I}_{S_{i}, X}, \mathscr{I}_{S_{j}, X}\right)=$ 0 for all $j<i$ and $\operatorname{Hom}_{X}\left(\mathscr{I}_{S_{i}, X}, \mathscr{I}_{S_{i}, X}\right) \cong \mathbf{k}$, we get that the sequence (4) splits, contradicting Lemma2.8,

Now without loss of generality, assume $e:=\left|N_{1}\right| \geq 2$. If $i \notin N_{1}$, then by permuting the first $i-1$ indices of $S_{j}$ 's we may assume $\mathscr{A}_{1} \in \llbracket\left(S_{1}, \ldots, S_{e}\right)$. Then by Lemma2.7we have $\operatorname{hom}_{X}\left(\mathscr{I}_{S_{j}, X}, \mathscr{A}_{1}\right)=\operatorname{hom}_{X}\left(\mathscr{A}_{1}, \mathscr{I}_{S_{i}, X}\right)=$ 0 for all $j \geq e+1$. Thus $f$ induces an isomorphism $f^{\prime}: \mathscr{A}_{1} \rightarrow \oplus_{j=1}^{e} \mathscr{I}_{S_{j}, X}$, contradicting the assumption $e \geq 2$ and the indecomposability of $\mathscr{A}_{1}$. If $i \in N_{1}$, then by permuting the first $i-1$ indices of $S_{j}$ 's we may assume $\mathscr{A}_{1} \in \llbracket\left(S_{i-e+1}, \ldots, S_{i}\right)$. From the case when $i \notin N_{1}$ we may also assume $\left|N_{j}\right|=1$ for all $j>1$, and this implies $\mathscr{J} \cong \mathscr{A}_{1} \oplus\left(\oplus_{j=1}^{i-e} \mathscr{I}_{S_{j}, X}\right)$. Then by Lemma2.7 we have $\operatorname{Hom}_{X}\left(\mathscr{I}_{S_{j}, X}, \mathscr{A}_{1}\right)=0$ for all $j \leq i-e$. In particular, the extension class $\varepsilon=\left(\varepsilon_{1}, \ldots, \varepsilon_{i-1}\right)$ corresponding to (4) with $\varepsilon_{j} \in \operatorname{Ext}_{X}^{1}\left(\mathscr{I}_{S_{i}, X}, \mathscr{I}_{S_{j}, X}\right)$ satisfies $\varepsilon_{j}=0$ for all $j \leq i-e$, contradicting Lemma2.8 and the generality of $\varepsilon$. 
Remark 2.11. As in the proof of Lemma 2.10 let us consider a general sheaf $\tilde{\mathscr{J}}$ fitting into an exact sequence

$$
0 \rightarrow \oplus_{j=1}^{i} \mathscr{I}_{S_{j}, X} \rightarrow \tilde{J} \rightarrow \mathscr{I}_{S_{0}, X} \rightarrow 0 .
$$

By Remark 2.9 the extension (5) is non-trivial. Here, $\tilde{\mathcal{J}} \in \mathbb{J}\left(S_{1}, \ldots, S_{i} ; S_{0}\right)$ and the sequence (5) is the Harder-Narasimhan filtration of $\tilde{\mathscr{J}}$. Assume that $\tilde{\mathscr{J}}$ is decomposable, say $\tilde{\mathscr{J}} \cong \tilde{\mathscr{A}}_{1} \oplus \cdots \oplus \tilde{\mathscr{A}}_{h}$. Note that the HN filtration of $\tilde{\mathscr{J}}$ is obtained from the ones of each $\tilde{\mathscr{A}}_{i}$. In particular, as in the proof of Lemma2.10, we have a partition $\left\{N_{j} \mid 1 \leq j \leq h\right\}$ of $\{0,1, \cdots, i\}$ such that $\tilde{\mathscr{A}}_{j} \in \mathbb{\square}\left(S_{k} ; k \in N_{j}\right)$ if $0 \notin N_{j}$, and $\tilde{\mathscr{A}}_{j} \in \mathbb{J}\left(S_{k} ; k \in\right.$ $N_{j} \backslash\{0\} ; S_{0}$ ). Then by the same argument in the proof of Lemma2.10, we get a contradiction. Thus we get an indecomposable sheaf in $\sqrt{ }\left(S_{1}, \ldots, S_{i} ; S_{0}\right)$.

Lemma 2.12. For each integer $i \geq 1$, the $\operatorname{set} \llbracket\left(S_{1}, \ldots, S_{i}\right)$ is parametrized by an affine space $T\left(S_{1}, \ldots, S_{i}\right)$, not necessarily finite-to-one, equipped with the universal sheaf, i.e. a sheaf $\mathscr{S}\left(S_{1}, \ldots, S_{i}\right)$ on $T\left(S_{1}, \ldots, S_{i}\right) \times X$ such that the fiber of $\mathscr{S}\left(S_{1}, \ldots, S_{i}\right)$ over $\{\mathscr{J}\} \times X$ with $\mathscr{J} \in \square\left(S_{1}, \ldots, S_{i}\right)$ is the sheaf $\mathscr{J}$ on $X$.

Proof. For $i=1$ we may take as $T\left(S_{1}\right)$ just a single point set, because $\square\left(S_{1}\right)=\left\{\mathscr{I}_{S_{1}, X}\right\}$. Assume that there exists an affine space $T\left(S_{1}, \ldots, S_{i-1}\right)$ and a sheaf $\mathscr{S}\left(S_{1}, \ldots, S_{i-1}\right)$ with prescribed property for $i \geq 2$. We set

$$
\begin{aligned}
T\left(S_{1}, \ldots, S_{i}\right) & :=\mathscr{E} x t_{p_{1}}^{1}\left(\mathscr{S}\left(S_{1}, \ldots, S_{i-1}\right), p_{2}^{*} \mathscr{I}_{S_{i}, X}\right) \\
& =R^{i}\left(p_{1_{*}} \not{H} \operatorname{om}_{T\left(S_{1}, \ldots, S_{i-1}\right) \times X}\left(\mathscr{S}\left(S_{1}, \ldots, S_{i-1}\right),-\right)\right)\left(p_{2}^{*} \mathscr{I}_{S_{i}, X}\right)
\end{aligned}
$$

to be the relative $\mathscr{E} x t_{p_{1}}^{1}$-sheaf, where $p_{j}$ is the projection from $T\left(S_{1}, \ldots, S_{i-1}\right) \times X$ to its $j$-th factor; see [20, Proposition 3.1]. By Lemma2.8 we have $\operatorname{ext}_{X}^{1}\left(\mathscr{J}^{\prime}, \mathscr{I}_{S_{i}, X}\right)=2 i-2$ for each $\mathscr{J}^{\prime} \in T\left(S_{1}, \ldots, S_{i-1}\right)$. This implies that $T\left(S_{1}, \ldots, S_{i}\right)$ is a vector bundle of rank $2 i-2$ over $T\left(S_{1}, \ldots, S_{i-1}\right)$ and so it is an affine space parametrizing $\square\left(S_{1}, \ldots, S_{i}\right)$ as required. We may also take as $\mathscr{S}\left(S_{1}, \ldots, S_{i}\right)$ the universal extension on $T\left(S_{1}, \ldots, S_{i}\right) \times X$ as in [20, Corollary 3.4].

Remark 2.13. Following the same argument in the proof of Lemma 2.12, we can obtain an affine space $\tilde{T}\left(S_{1}, \ldots, S_{i} ; S_{0}\right)$ parametrizing $\mathbb{J}\left(S_{1}, \ldots, S_{i}\right)$ equipped with the universal sheaf $\tilde{\mathscr{S}}\left(S_{1}, \ldots, S_{i} ; S_{0}\right)$.

Proof of Theorem 2.4: Assume that $r$ is even and set $m:=r / 2$. Fix subsets $S_{1}, \ldots, S_{m} \subset X_{\text {reg }}$ such that $\left|S_{i}\right|=2$ for all $i$ and $S_{i} \cap S_{j}=\varnothing$ for all $i \neq j$. By Lemma 2.10 there exists an indecomposable sheaf $\mathscr{J} \in$ $\llbracket\left(S_{1}, \ldots, S_{m}\right)$, for which we consider a general sheaf $\mathscr{E}$ fitting into the following exact sequence:

$$
0 \rightarrow \mathscr{O}_{X}^{\oplus m} \stackrel{f}{\rightarrow} \mathscr{E} \rightarrow \mathscr{J} \rightarrow 0 .
$$

Note that $\mathscr{E}$ has rank $r$ with $\operatorname{det}(\mathscr{E}) \cong \mathscr{O}_{X}$ and $c_{2}(\mathscr{E})=r$. Let $\varepsilon=\left(\varepsilon_{1}, \ldots, \varepsilon_{m}\right) \in \operatorname{Ext}_{X}^{1}\left(\mathscr{J}, \mathscr{O}_{X}^{\oplus m}\right)$ be the extension class corresponding to [6] with $\varepsilon_{i} \in \operatorname{Ext}_{X}^{1}\left(\mathscr{J}, \mathscr{O}_{X}\right)$. Note that $h^{0}(\mathscr{E})=m$ and $f\left(\mathscr{O}_{X}^{\oplus m}\right)$ is the image of the evaluation map $\rho_{\mathscr{E}}: H^{0}(\mathscr{E}) \otimes \mathscr{O}_{X} \rightarrow \mathscr{E}$ with $\mathscr{J}=\operatorname{coker}\left(\rho_{\mathscr{E}}\right)$.

By Lemma 2.5 and Serre's duality, we have $\operatorname{ext}_{X}^{1}\left(\mathscr{J}, \mathscr{O}_{X}\right)=h^{1}(\mathscr{J})=m$. From the generality of $\varepsilon$ we see that the extensions $\varepsilon_{1}, \ldots, \varepsilon_{m}$ are linearly independent. In particular, we have $A \cdot \varepsilon \neq 0$ for all $A \in \operatorname{GL}(m)$, and so $\mathscr{E} \not \mathscr{O}_{X} \oplus \mathscr{G}$ with $\mathscr{G}$ an extension of $\mathscr{J}$ by $\mathscr{O}_{X}^{\oplus(m-1)}$. Since $f\left(\mathscr{O}_{X}^{\oplus m}\right) \subset \mathscr{E}$ is the image of $\rho_{\mathscr{E}}$, we get that $\mathscr{E} \not \mathscr{O}_{X} \oplus \mathscr{G}$ for any sheaf $\mathscr{G}$, i.e. $\mathscr{E}$ has no trivial factor.

Assume that $\mathscr{E}$ is decomposable, say $\mathscr{E} \cong \mathscr{E}_{1} \oplus \mathscr{E}_{2}$ with each $\mathscr{E}_{i} \neq 0$. Since the global section functor $H^{0}(-)$ and the evaluation map commute with direct sums, we have $\mathscr{J} \cong \operatorname{coker}\left(\rho_{\mathscr{E}_{1}}\right) \oplus \operatorname{coker}\left(\rho_{\mathscr{E}_{2}}\right)$. Since $\mathscr{J}$ is indecomposable, we get $\operatorname{coker}\left(\rho_{\mathscr{E}_{i}}\right)=0$ for some $i \in\{1,2\}$. This implies that $\mathscr{E}_{i}$ is trivial, which is impossible because $\mathscr{E}$ has no trivial factor.

To conclude the case $r$ even we need to find a sheaf $\mathscr{E}$ that is locally free and aCM. Consider the variety $T\left(S_{1}, \ldots, S_{m}\right)$ together with the sheaf $\mathscr{S}\left(S_{1}, \ldots, S_{m}\right)$ in Lemma2.12. Define

$$
\mathcal{V}\left(S_{1}, \ldots, S_{m}\right):=\mathscr{E} x t_{p_{2}}^{1}\left(\mathscr{S}\left(S_{1}, \ldots, S_{m}\right), p_{2}^{*} \mathscr{O}_{X}^{\oplus m}\right)
$$


to be the relative $\mathscr{E} x t_{p_{2}}^{1}$-sheaf as in [20, Proposition 3.1]; the fibre of $\mathcal{V}\left(S_{1}, \ldots, S_{m}\right)$ over a point $\mathscr{J} \in$ $T\left(S_{1}, \ldots, S_{m}\right)$ is the set of all extensions of $\mathscr{J}$ by $\mathscr{O}_{X}^{\oplus m}$. By Lemma 2.5 the sheaf $\mathcal{V}\left(S_{1}, \ldots, S_{m}\right)$ is a vector bundle of rank $m^{2}$ on $T\left(S_{1}, \ldots, S_{m}\right)$ and so it is an affine space. Since $\mathscr{G}_{S_{1}} \oplus \cdots \oplus \mathscr{G}_{S_{m}}$ is locally free and $\mathrm{aCM}$, the sheaf associated to a general point in $\mathcal{V}$ is locally free and aCM. Define

$$
\mathbb{U}:=\left\{\left(S_{1}, \ldots, S_{m}\right) \mid S_{i} \subset X_{\text {reg }} \text { with }\left|S_{i}\right|=2 \text { and } S_{i} \cap S_{j}=\varnothing \text { for all } i \neq j\right\}
$$

and consider a vector bundle $\mathcal{V}$ on $\mathbb{U}$, whose fibre over $\left(S_{1}, \ldots, S_{m}\right)$ is $\mathcal{V}\left(S_{1}, \ldots, S_{m}\right)$. Then there exists a non-empty open subset $V^{\prime} \subset \mathcal{V}$ such that the middle term of each extension in $V^{\prime}$ is aCM and locally free. As in the proof of Theorem 2.3 we can choose an irreducible subvariety $\Gamma \subset V^{\prime}$ such that the restriction of the map $V^{\prime} \rightarrow \mathbb{U}$ to $\Gamma$ is quasi-finite and dominant. Hence we get the assertion for the case $r$ even.

Now assume that $r$ is odd, say $r=2 m+3$. The case $m=0$ is true by Proposition 2.1 with $r=3$, because we have $g=h^{0}\left(\mathscr{O}_{X}(1)\right) \geq 3$. Now assume $r \geq 5$, i.e. $m \geq 1$, and that Theorem 2.4 is true for all odd integers less than $r$. We fix subsets $S_{0}, \ldots, S_{m} \subset X_{\text {reg }}$ with $\left|S_{0}\right|=3$ and $\left|S_{i}\right|=2$ for all $i \geq 1$ such that $S_{i} \cap S_{j}=\varnothing$ for all $i \neq j$. Define

$$
\mathscr{W}\left(S_{1}, \ldots, S_{m} ; S_{0}\right):=\mathscr{E} x t_{p_{2}}^{1}\left(\tilde{\mathscr{S}}\left(S_{1}, \ldots, S_{m} ; S_{0}\right), p_{2}^{*} \mathscr{O}_{X}^{\oplus(m+2)}\right),
$$

where $\tilde{\mathscr{S}}\left(S_{1}, \ldots, S_{m} ; S_{0}\right)$ is the universal sheaf in Remark 2.13. Then it parametrizes all the extensions of some sheaf $\tilde{\mathscr{J}} \in \mathbb{J}\left(S_{1}, \ldots, S_{m} ; S_{0}\right)$ by $\mathscr{O}_{X}^{\oplus(m+2)}$. Note that for each extension in $\mathbb{W}\left(S_{1}, \ldots, S_{m} ; S_{0}\right)$ the corresponding middle term $\mathscr{E}$ is torsion-free and has rank $r=2 m+3$ with $\operatorname{det}(\mathscr{E}) \cong \mathscr{O}_{X}$ and $c_{2}(\mathscr{E})=r$.

Let us denote by $\mathscr{G}_{S_{0}}$ an aCM and indecomposable vector bundle of rank three, admitting an extension of $\mathscr{I}_{S_{0}, X}$ by $\mathscr{O}_{X}^{\oplus 2}$ as in Proposition 2.1 . Then $\oplus_{i=1}^{m} \mathscr{G}_{S_{i}}$ is the middle term of an extension in $\mathbb{W}\left(S_{1}, \ldots, S_{m} ; S_{0}\right)$, which is locally free and aCM. So the general extension in $\mathbb{W}\left(S_{1}, \ldots, S_{m} ; S_{0}\right)$ has the aCM and indecomposable middle term. Now fix an indecomposable sheaf $\tilde{\mathcal{J}} \in \mathbb{J}\left(S_{1}, \ldots, S_{m} ; S_{0}\right)$ in Remark 2.11 and consider a general sheaf $\mathscr{E}$ fitting into the following exact sequence:

$$
0 \rightarrow \mathscr{O}_{X}^{\oplus(m+2)} \stackrel{f}{\rightarrow} \stackrel{g}{\mathscr{E}} \stackrel{g}{\rightarrow} \tilde{J} \rightarrow 0 .
$$

Assume that $\mathscr{E}$ is decomposable, say $\mathscr{E} \cong \mathscr{E}_{1} \oplus \mathscr{E}_{2}$ with each $\mathscr{E}_{i} \not \approx 0$. As before, $f\left(\mathscr{O}_{X}^{\oplus(m+2)}\right)$ is the image of the evaluation map $\rho_{\mathscr{E}}: H^{0}(\mathscr{E}) \otimes \mathscr{O}_{X} \rightarrow \mathscr{E}$ and $\operatorname{coker}\left(\rho_{\mathscr{E}}\right)=\tilde{\mathscr{J}}$. Since the global section functor $H^{0}(-)$ and the evaluation map commute with finite direct sums, we have $\tilde{\mathscr{J}} \cong \operatorname{coker}\left(\rho_{\mathscr{E}_{1}}\right) \oplus \operatorname{coker}\left(\rho_{\mathscr{E}_{2}}\right)$. Since $\tilde{\mathscr{J}}$ is indecomposable, we get that $\mathscr{E}_{i}$ is trivial for some $i$, which contradicts to the generality of the extension (7), because we have $\operatorname{ext}_{X}^{1}\left(\tilde{\mathscr{J}}, \mathscr{O}_{X}\right)=h^{1}(\tilde{\mathscr{J}})=m+2$ by Remark 2.6. As in the case $r$ even, we define

$$
\begin{aligned}
\tilde{\mathbb{U}}:=\left\{\left(S_{0}, S_{1}, \ldots, S_{m}\right) \mid\right. & S_{i} \subset X_{\text {reg with }\left|S_{0}\right|=3,} \\
& \left.\left|S_{i}\right|=2 \text { for all } 1 \leq i \leq m \text { and } S_{i} \cap S_{j}=\varnothing \text { for all } i \neq j\right\} .
\end{aligned}
$$

We consider a vector bundle $\mathbb{W}$ on $\mathbb{U}$, whose fibre over $\left(S_{0}, S_{1}, \ldots, S_{m}\right)$ is $\mathbb{W}\left(S_{1}, \ldots, S_{m} ; S_{0}\right)$. Then we get the assertion, following the same argument in the case $r$ even.

Remark 2.14. Let $\pi: Y \rightarrow X$ be a birational morphism between integral projective surfaces with $\omega_{X} \cong$ $\mathscr{O}_{X}$ and $q(X)=0$ such that $\pi$ induces an isomorphism $\pi^{-1}\left(X_{\text {sing }}\right) \cong X_{\text {sing. }}$. In particular, we have $Y_{\text {reg }}=$ $\pi^{-1}\left(X_{\text {reg }}\right)$. This implies that $\pi_{*} \mathscr{O}_{Y} \cong \mathscr{O}_{X}$ and $R^{1} \pi_{*} \mathscr{O}_{Y} \cong 0$. Since each fiber of $\pi$ has dimension at most one, we also have $R^{2} \pi_{*} \mathscr{F} \cong 0$ for any coherent sheaf $\mathscr{F}$ on $X$. Thus we have $q(Y)=0$ and $h^{2}\left(\mathscr{O}_{Y}\right)=1$. Since $\pi$ induces an isomorphism between $\pi^{-1}\left(X_{\text {sing }}\right)$ and $X_{\text {sing }}$, the canonical sheaf $\omega_{Y}$ is locally free with $h^{0}\left(\omega_{Y}\right)=1$ and so there is an effective divisor $\Delta$ such that $\left|\omega_{Y}\right|=\{\Delta\}$; we have $\Delta=\varnothing$ if and only if $\pi$ is an isomorphism. By Serre's duality we have $\operatorname{ext}_{Y}^{1}\left(\mathscr{I}_{S, Y}, \mathscr{O}_{Y}\right)=h^{1}\left(\mathscr{I}_{S, Y} \otimes \omega_{Y}\right)$. Since $\left|\omega_{Y}\right|=\{\Delta\}$ and $S \cap \Delta=\varnothing$, we may use the long exact sequence of cohomology of the following exact sequence

$$
0 \rightarrow \mathscr{I}_{S, Y} \otimes \omega_{Y} \rightarrow \omega_{Y} \rightarrow \mathscr{O}_{S} \rightarrow 0
$$

to obtain $\operatorname{ext}_{Y}^{1}\left(\mathscr{I}_{S, Y}, \mathscr{O}_{Y}\right)=|S|-1$ for any finite subset $S \subset Y_{\text {reg }} \backslash \Delta$. Then the same statement of Theorem 2.4 holds for $Y$, using the same argument in its proof with subsets $S_{i} \subset Y_{\text {reg }} \backslash \Delta$ for $i=0, \cdots, m$. 


\section{ENRIQUES SURFACES}

In this section we assume that $X$ is an integral projective surface with $q(X)=0$ and $\omega_{X} \nsucceq \mathscr{O}_{X}$ locally free such that $h^{0}\left(\omega_{X}\right)=0$ and $h^{0}\left(\omega_{X}^{\otimes 2}\right)=1$. Let $\Delta \geq 0$ be the effective divisor such that $\omega_{X}^{\otimes 2} \cong \mathscr{O}_{X}(\Delta)$. When $X$ is smooth, the minimal model of $X$ is an Enriques surface. Note that $h^{2}\left(\mathscr{O}_{X}\right)=h^{0}\left(\omega_{X}\right)=0$ and so $\chi\left(\mathscr{O}_{X}\right)=1$. Set $X^{\prime}:=X_{\text {reg }} \cap(X \backslash \Delta)$.

Remark 3.1. We fix an ample line bundle $\mathscr{O}_{X}(1)$ on $X$ such that $h^{1}\left(\mathscr{O}_{X}(t)\right)=0$ for all $t \in \mathbb{Z}$; at least in characteristic zero Kodaira's vanishing theorem shows that we only need this assumption for $t \geq 0$. The case $t=0$ is a general assumption of the surfaces considered in this article. Serre's duality gives $h^{1}\left(\omega_{X}(t)\right)=0$ for all $t \in \mathbb{Z}$. Notice that using Riemann-Roch it is easy to see that under these hypothesis $h^{0}\left(\omega_{X}(1)\right) \neq 0$. In summary, we take a polarization $\mathscr{O}_{X}(1)$ such that $h^{0}\left(\omega_{X}(1)\right) \neq 0$ and $h^{1}\left(\mathscr{O}_{X}(t)\right)=h^{1}\left(\omega_{X}(t)\right)=0$ for all $t \in \mathbb{Z}$. If $\Delta=\varnothing$, e.g. minimal Enriques surfaces, then we always have $h^{1}\left(\mathscr{O}_{X}(t)\right)=0$ for $t>0$, because $\omega_{X}(t)$ with $t>0$ is ample; it is numerically equivalent to $\mathscr{O}_{X}(t)$ and so we can use Kodaira's vanishing theorem.

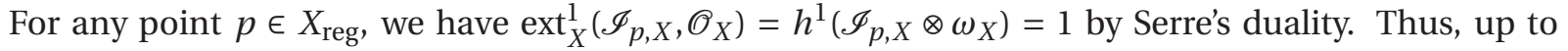
isomorphisms, there is a unique sheaf $\mathscr{E}_{p}$ that fits into the following non-trivial extension:

$$
0 \rightarrow \mathscr{O}_{X} \rightarrow \mathscr{E}_{p} \rightarrow \mathscr{I}_{p, X} \rightarrow 0 .
$$

Obviously $\mathscr{E}_{p}$ has rank two and it is locally free outside $p$ with $\operatorname{det}\left(\mathscr{E}_{p}\right) \cong \mathscr{O}_{X}$. Since $p \in X_{\text {reg }}$ and $h^{0}\left(\omega_{X}\right)=$ 0 , the Cayley-Bacharach condition is satisfied. Thus $\mathscr{E}_{p}$ is locally free. Note that the point $p$ is uniquely determined by the isomorphism class of $\mathscr{E}_{p}$, because we have $h^{0}\left(\mathscr{E}_{p}\right)=1$ by the sequence (8) and any non-zero section of $\mathscr{E}_{p}$ vanishes only at $p$.

Lemma 3.2. For a general $p \in X_{\text {reg }}$ the vector bundle $\mathscr{E}_{p}$ is aCM and indecomposable.

Proof. The exact sequence (8) twisted by $\mathscr{O}_{X}(t)$ gives $h^{1}\left(\mathscr{E}_{p}(t)\right)=0$ for all $t \geq 0$. From $\mathscr{E}_{p}^{\vee} \cong \mathscr{E}_{p}$ we see that $h^{1}\left(\mathscr{E}_{p} \otimes \omega_{X}\right)=h^{1}\left(\mathscr{E}_{p}\right)=0$ by Serre's duality. Now fix an integer $t<0$. The twist of the sequence (8) by $\omega_{X}(-t)$ gives

$$
h^{1}\left(\mathscr{E}_{p} \otimes \omega_{X}(-t)\right) \leq h^{1}\left(\omega_{X}(-t)\right)+h^{1}\left(\mathscr{I}_{p, X} \otimes \omega_{X}(-t)\right)=h^{1}\left(\mathscr{I}_{p, X} \otimes \omega_{X}(-t)\right) .
$$

Here, we have $h^{1}\left(\omega_{X}(-t)\right)=0$ by our assumptions on the polarization $\mathscr{O}_{X}$. We also have $h^{0}\left(\omega_{X}(-t)\right)>0$ from the assumption that $h^{0}\left(\omega_{X}(1)\right)>0$. Since $p$ is general, we have $h^{1}\left(\mathscr{I}_{p, X} \otimes \omega_{X}(-t)\right)=0$. By Serre's duality, this implies that $h^{1}\left(\mathscr{E}_{p}(t)\right)=h^{1}\left(\mathscr{E}_{p} \otimes \omega_{X}(-t)\right)=0$. Thus $\mathscr{E}_{p}$ ia aCM.

Assume that $\mathscr{E}_{p}$ is decomposable; say $\mathscr{E}_{p} \cong \mathscr{A}_{1} \oplus \mathscr{A}_{2}$ with each $\mathscr{A}_{i}$ a line bundle. Since $h^{0}\left(\mathscr{E}_{p}\right)=1$, we may assume that $h^{0}\left(\mathscr{A}_{1}\right)=1$ and $h^{0}\left(\mathscr{A}_{2}\right)=0$. Since the evaluation map commutes with direct sums and $\mathscr{I}_{p, X}$ is isomorphic to the cokernel of the evaluation map $H^{0}\left(\mathscr{E}_{p}\right) \otimes \mathscr{O}_{X} \rightarrow \mathscr{E}_{p}$, we get $\mathscr{A}_{2} \cong \mathscr{I}_{p, X}$, a contradiction.

Lemma 3.3. For any two general points $p, q \in X_{\mathrm{reg}}$, we have $\operatorname{ext}_{X}^{1}\left(\mathscr{E}_{p}, \mathscr{E}_{q}\right)=1$.

Proof. Since $\operatorname{det}\left(\mathscr{E}_{q}\right) \cong \mathscr{O}_{X}$, we have $\mathscr{E}_{q}^{\vee} \cong \mathscr{E}_{q}$ and so $\operatorname{Ext}_{X}^{1}\left(\mathscr{E}_{p}, \mathscr{E}_{q}\right) \cong H^{1}\left(\mathscr{E}_{p} \otimes \mathscr{E}_{q}\right)$. Tensoring the exact sequence (8) with $\mathscr{E}_{q}$, we get the exact sequence

$$
0 \rightarrow \mathscr{E}_{q} \rightarrow \mathscr{E}_{p} \otimes \mathscr{E}_{q} \rightarrow \mathscr{I}_{p, X} \otimes \mathscr{E}_{q} \rightarrow 0 .
$$

Since $\mathscr{E}_{q}$ is aCM, we have $h^{1}\left(\mathscr{E}_{q}\right)=0$. On the other hand, tensoring the sequence (8) for $\mathscr{E}_{q}$ with $\omega_{X}$ gives $h^{0}\left(\mathscr{E}_{q} \otimes \omega_{X}\right)=0$, because $\omega_{X} \not \approx \mathscr{O}_{X}$. Thus by Serre's duality we get $h^{2}\left(\mathscr{E}_{q}\right)=h^{0}\left(\mathscr{E}_{q} \otimes \omega_{X}\right)=0$. Then the assertion follows from the exact sequence

$$
0 \rightarrow \mathscr{I}_{p, X} \otimes \mathscr{E}_{q} \rightarrow \mathscr{E}_{q} \rightarrow\left(\mathscr{E}_{q}\right)_{\mid\{p\}} \rightarrow 0
$$

together with the fact that $\mathscr{E}_{q}$ is an aCM vector bundle of rank two and $H^{0}\left(\mathscr{E}_{q}\right)$ is one-dimensional whose nontrivial section vanishes only at $q$ so that $h^{0}\left(\mathscr{I}_{p, X} \otimes \mathscr{E}_{q}\right)=0$. 
Proposition 3.4. Setting $\tilde{g}:=h^{0}\left(\mathscr{O}_{X}(1)\right)$, there exists an indecomposable aCM vector bundle $\mathscr{E}$ of rank $r$ on $X$ with $\operatorname{det}(\mathscr{E}) \cong \mathscr{O}_{X}$ and $c_{2}(\mathscr{E})=r-1$ for each integer $2 \leq r \leq \tilde{g}-1$.

Proof. As in the proof of Proposition 2.1, consider a general sheaf $\mathscr{E}$ fitting into the sequence (1) for a general $S \subset X_{\text {reg }}$ with $|S|=r-1$. Then we get $\operatorname{ext}_{X}^{1}\left(\mathscr{I}_{S, X}, \mathscr{O}_{X}\right)=r-1$ and the proof of Proposition 2.1 works verbatim.

Theorem 3.5. Let $X$ be an integral projective surface with $q(X)=0$ and $\omega_{X} \nsucceq \mathscr{O}_{X}$ locally free such that $h^{0}\left(\omega_{X}\right)=0$ and $h^{0}\left(\omega_{X}^{\otimes 2}\right)=1$. Then for any $r \geq 2$ there exists a family $\left\{\mathscr{E}_{\alpha}\right\}_{\alpha \in \Gamma}$ of dimension $2\left\lceil\frac{r}{2}\right\rceil$ of indecomposable rank $r$ aCM vector bundles with $c_{1}\left(\mathscr{E}_{\alpha}\right) \cong \mathscr{O}_{X}$ such that for each $\alpha \in \Gamma$ there are only finitely many $\beta \in \Gamma$ with $\mathscr{E}_{\beta} \cong \mathscr{E}_{\alpha}$.

Proof. The proof follows exactly the same structure as in the case of Theorem 2.4. In the present setting, however, in the case of even rank $r=2 \mathrm{~m}$, the family $\Gamma$ of indecomposable aCM vector bundles of rank $r$ will be mapped by a quasi-finite dominant morphism to

$$
\mathbb{U}:=\left\{\left(S_{1}, \ldots, S_{m}\right) \mid S_{i} \subset X_{\text {reg }} \text { with }\left|S_{i}\right|=1 \text { and } S_{i} \cap S_{j}=\varnothing \text { for all } i \neq j\right\},
$$

a variety of dimension $r$, while in the odd case $r=2 m+3$ it will be mapped to

$$
\begin{aligned}
\widetilde{\mathbb{U}}:=\left\{\left(S_{0}, S_{1}, \ldots, S_{m}\right) \mid\right. & S_{i} \subset X_{\text {reg }} \text { with }\left|S_{0}\right|=2, \\
& \left.\left|S_{i}\right|=1 \text { for all } 1 \leq i \leq m \text { and } S_{i} \cap S_{j}=\varnothing \text { for all } i \neq j\right\} .
\end{aligned}
$$

a variety of dimension $2 m+4=2\left\lceil\frac{r}{2}\right\rceil$.

\section{IRREGULAR SURFACES}

In this section we deal with surfaces with $q(X) \geq 1$.

Proposition 4.1. Let $X$ be a smooth projective surface with $q(X)=1$ and a fixed ample line bundle $\mathscr{O}_{X}(1)$, satisfying one of the following conditions:

(i) $\mathscr{O}_{X}(1) \cong \omega_{X}$;

(ii) $\mathscr{O}_{X}(1) \otimes \omega_{X}^{\vee}$ is ample.

Then for each positive integer $r$ there exists a one-dimensional family $\left\{\mathscr{E}_{\alpha}\right\}_{\alpha \in \Gamma}$ of indecomposable aCM vector bundles of rank $r$ on $X$ such that $\mathscr{E}_{\alpha}$ for each $\alpha \in \Gamma$ is strictly semistable with $\operatorname{det}\left(\mathscr{E}_{\alpha}\right) \in \operatorname{Pic}^{0}(X)$ and $c_{2}\left(\mathscr{E}_{\alpha}\right)=0$ with respect to any polarization of $X$, and there are only finitely many $\beta \in \Gamma$ with $\mathscr{E}_{\beta} \cong \mathscr{E}_{\alpha}$.

Proof. Fix a general line bundle $\mathscr{L} \in \operatorname{Pic}^{0}(X)$. Then we have $h^{1}(\mathscr{L})=0$; see [3, Th. 0.1], [12, Theorem 1] or [13, Theorem 0.1]. We also have $h^{1}(\mathscr{L}(-t))=0$ for all $t>0$ by Kodaira's vanishing. Note that Serre's duality gives $h^{1}(\mathscr{L}(t))=h^{1}\left(\mathscr{L}^{\vee} \otimes \omega_{X}(-t)\right)$. Then we have $h^{1}\left(\mathscr{L}^{\vee} \otimes \omega_{X}(-t)\right)=0$ for all $t>0$. Indeed, in case (i) we may apply Kodaira's vanishing for $t \geq 2$ and $h^{1}\left(\mathscr{L}^{\vee}\right)=0$ for $t=1$. In case (ii) $\omega_{X}^{\vee}(t)$ is ample and so we may apply Kodaira's vanishing. Thus $\mathscr{L}$ is aCM.

Let $\varphi: X \rightarrow C$ be the Albanese map of $X$ onto an elliptic curve $C$. We have $\varphi_{*} \mathscr{O}_{X} \cong \mathscr{O}_{C}$ and $\operatorname{Pic}^{0}(X)=$ $\varphi^{*} \operatorname{Pic}(C)$. By the classification of vector bundles on an elliptic curve in [1], there is an indecomposable vector bundle $\mathscr{F}$ of rank $r$ on $C$, which is an iterated extension of $\mathscr{O}_{C}$. Define

$$
\mathscr{E}_{\mathscr{L}}:=\varphi^{*} \mathscr{F} \otimes \mathscr{L} .
$$

Then $\mathscr{E} \mathscr{L}$ is a vector bundle of rank $r$ on $X$ with $\operatorname{det}(\mathscr{E} \mathscr{L}) \cong \mathscr{L}^{\otimes r} \in \operatorname{Pic}^{0}(X)$ and $c_{2}(\mathscr{E} \mathscr{L})=0$, which is an iterated extension of $\mathscr{L}$. Since $\mathscr{L}$ is aCM, so is $\mathscr{E} \mathscr{L}$.

Assume that $\mathscr{E}_{\mathscr{L}}$ is decomposable and this would imply that $\varphi^{*} \mathscr{F}$ is also decomposable, say $\varphi^{*} \mathscr{F} \cong$ $\mathscr{F}_{1} \oplus \mathscr{F}_{2}$ with each $\mathscr{F}_{i}$ an aCM vector bundle of rank $r_{i}$ with $0<r_{i}<r$. By the projection formula and $\varphi_{*} \mathscr{O}_{X} \cong \mathscr{O}_{C}$, we have $\mathscr{F} \cong \varphi_{*} \mathscr{F}_{1} \oplus \varphi_{*} \mathscr{F}_{2}$. Now take a non-empty subset of $C$ so that

- we have $\mathscr{F}_{\mid U} \cong \mathscr{O}_{U}^{\oplus r}$, and

- $\varphi^{-1}(q)$ is a smooth projective curve for each $q \in U$. 
Since $\left(\varphi^{*} \mathscr{F}\right)_{\mid \varphi^{-1}(q)}$ is the trivial vector bundle of rank $r$ on the integral projective curve $\varphi^{-1}(q)$, we get $\mathscr{F}_{i \mid \varphi^{-1}(q)} \cong \mathscr{O}_{\mid \varphi^{-1}(q)}^{\oplus r_{i}}$ for each $i$. In particular, we have $\varphi_{*} \mathscr{F}_{i}$ is not zero for each $i$, a contradiction to the indecomposability of $\mathscr{F}$.

Remark 4.2. Let $X$ be a smooth and connected projective variety of dimension $n \geq 2$ and $\varphi: X \rightarrow \operatorname{Alb}(X)$ its Albanese map. Assume that $X$ has maximal Albanese dimension, i.e. $\operatorname{dim} \varphi(X)=n$. Note that this implies $q(X)=\operatorname{dim} \operatorname{Alb}(X)=n \geq 2$. In particular, an abelian variety has maximal Albanese dimension. Let $\mathscr{O}_{X}(1)$ be an ample line bundle on $X$ such that $\omega_{X}^{\vee} \otimes \mathscr{O}_{X}(1)$ is ample; if $X$ is an abelian variety, then $\mathscr{O}_{X}(1)$ can be arbitrary.

Now choose a general line bundle $\mathscr{L} \in \operatorname{Pic}^{0}(X)$. Since $X$ has Albanese dimension $n$, we have $h^{i}(\mathscr{L})=$ 0 for all $1 \leq i \leq n-1$ by [12, Theorem 1] or [13, Theorem 0.1]. Fix a positive integer $t$. By Kleiman's numerical criterion of ampleness in [17], we get that $\mathscr{L}^{\vee}(t)$ and $\omega_{X}^{\vee} \otimes \mathscr{L}(t)$ are ample for $t>0$. Then Kodaira's vanishing gives $h^{i}(\mathscr{L}(-t))=h^{i}\left(\omega_{X} \otimes \mathscr{L}^{\vee}(-t)\right)=0$ for all $1 \leq i \leq n-1$. On the other hand, Serre's duality gives $h^{i}(\mathscr{L}(t))=h^{n-i}\left(\omega_{X} \otimes \mathscr{L}^{\vee}(-t)\right)=0$ for $1 \leq i \leq n-1$. This implies that $\mathscr{L}$ is aCM. Since $\operatorname{dim} \operatorname{Pic}^{0}(X)=q(X)$, there exists a $n$-dimensional family of pairwise non-isomorphic aCM lines bundles.

Now we work on the proof of Theorem 1.3 and the key tool is Mukai's study of vector bundles on abelian varieties; see [21].

Proof of Theorem 1.3 . Since $X$ is smooth and birational to an abelian variety, there are an $n$-dimensional abelian variety $Y$ and a proper birational morphism $v: X \rightarrow Y$; see [23. Proposition 9.12]. In particular, we have $v_{*} \mathscr{O}_{X} \cong \mathscr{O}_{Y}$ by the Zariski Main Theorem in [14, Corollary III.11.4]). Let $\widehat{Y}=\operatorname{Pic}^{0}(Y)$ denote the abelian variety dual to $Y$. As in [21, Definitions 4.4, 4.5, 4.6] we consider the following set

$$
\mathbb{U}_{r}^{\prime}:=\{\text { the unipotent vector bundles of rank } r \text { on } Y\} \text {, }
$$

i.e. the set of all vector bundles of rank $r$ on $Y$, obtained by $(r-1)$-times of iteration of $\mathscr{O}_{Y}$; we have $\mathbb{U}_{1}^{\prime}=\left\{\mathscr{O}_{Y}\right\}$ and $\mathbb{U}_{r}^{\prime}$ is the set of all vector bundles which admit extensions of $\mathscr{O}_{Y}$ by an element of $\mathbb{U}_{r-1}^{\prime}$. If we let $R$ be the completion of the local ring $\mathscr{O}_{\widehat{Y}, 0}$ and $B_{f}$ the set of all $R$-modules with finite length, then by [21, Theorem 4.12] there is a bijection between $\mathbb{U}_{r}^{\prime}$ and the set $B_{f}[r]$ of the $R$-modules of length $r$. Note that this bijection preserves finite direct sums. Thus to an indecomposable vector bundle in $\mathbb{U}_{r}^{\prime}$ it is enough to consider an indecomposable elements of $B_{f}[r]$. Define a subset

$$
\mathbb{U}_{r}:=\left\{\begin{array}{c|c}
\mathscr{A} \in \mathbb{U}_{r}^{\prime} & \begin{array}{c}
\mathscr{A} \text { corresponds to an indecomposable elements of } B_{f}[r] \\
\text { of the form } R / I \text { with } I \subset R \text { an ideal of colength } r
\end{array}
\end{array}\right\},
$$

consisting of elements of the local Hilbert scheme of $R$ corresponding to connected zero-dimensional subschemes of $\widehat{Y}$ of degree $r$ with 0 as their support. Then we get an algebraic family $\mathbb{U}_{r}$ of indecomposable unipotent vector bundles of rank $r$. For the known results on the dimension of $\mathbb{U}_{r}$, refer to [11, page 6]. For $n=2$ and arbitrary $r, \mathbb{U}_{r}$ is irreducible of dimension $r-1$ by [4, 16], while it can be reducible for $n \geq 3$ by [11, 16]. In any case with $n \geq 2, \mathbb{U}_{r}$ has an irreducible family of dimension $(n-1)(r-1)$, whose general element is curvilinear, or collinear, by [11, pages 5-6].

For any line bundle $\mathscr{L} \in \operatorname{Pic}^{0}(X)$, set

$$
\Theta_{\mathscr{L}}:=\left\{v^{*}(\mathscr{F}) \otimes \mathscr{L} \mid \mathscr{F} \in \mathbb{U}_{r}\right\} .
$$

Each element of $\Theta_{\mathscr{L}}$ is a vector bundle of rank $r$ on $X$, which is an iterated extension of $\mathscr{L}$. Thus each element of $\Theta_{\mathscr{L}}$ is strictly semistable with respect to any polarization on $X$ and all its Chern classes are zero. Assume that $v^{*}(\mathscr{F}) \otimes \mathscr{L} \cong v^{*}(\mathscr{G}) \otimes \mathscr{L}$ for $\mathscr{F}, \mathscr{G} \in \mathbb{U}_{r}$. Then we get $v^{*}(\mathscr{F}) \cong v^{*}(\mathscr{G})$ and so $\mathscr{F} \cong \mathscr{G}$ by the projection formula and $v_{*} \mathscr{O}_{X} \cong \mathscr{O}_{Y}$. In particular, $\Theta \mathscr{L}$ parametrizes one-to-one vector bundles of rank $r$ on $X$ and $\operatorname{dim} \Theta \mathscr{L}=\operatorname{dim} \mathbb{U}_{r}$. Note that for each $\mathscr{A} \in \Theta_{\mathscr{L}}$ there are only finitely many $\mathscr{L}^{\prime} \in \operatorname{Pic}^{0}(X)$ such that $\mathscr{A} \cong \mathscr{A}^{\prime}$ for some $\mathscr{A}^{\prime} \in \Theta \mathscr{L}^{\prime}$; indeed, we have at most $(2 n)^{r}$ vector bundles $\mathscr{A}^{\prime}$, because $\operatorname{det}(\mathscr{A}) \cong \mathscr{L}^{\otimes r}$ and so $\mathscr{L}^{\prime} \otimes \mathscr{L}^{\vee}$ is an element of $r$-torsion of $\operatorname{Pic}^{0}(X)$. Now a general line bundle $\mathscr{L} \in \operatorname{Pic}^{0}(X)$ is aCM by 
Remark 4.2, Define a non-empty open subset

$$
\mathbb{V}:=\left\{\mathscr{L} \in \operatorname{Pic}^{0}(X) \mid \mathscr{L} \text { is } \operatorname{aCM}\right\},
$$

which is an algebraic variety of dimension $q(X)=n$. For each $\mathscr{L} \in \mathbb{V}$, every vector bundle $\mathscr{A} \in \Theta_{\mathscr{L}}$ is aCM, because it is an iterated extension of aCM vector bundles. Define a parameter space $\Gamma$ over $\mathbb{V}$ whose fibre over $\mathscr{L}$ is $\Theta_{\mathscr{L}}$. Then it is a parameter space, finite-to-one, for indecomposable aCM vector bundles of $\operatorname{rank} r$ on $X$ with $\operatorname{dim} \Gamma=n+\operatorname{dim} \mathbb{U}_{r}=(n-1) r+1$.

Proposition 4.3. Let $X$ be a smooth projective surface with $q(X) \geq 2$ and a fixed ample line bundle $\mathscr{O}_{X}(1)$ satisfying one of the following conditions:

(i) $\mathscr{O}_{X}(1) \cong \omega_{X}$;

(ii) $\mathscr{O}_{X}(1) \otimes \omega_{X}^{\vee}$ is ample.

Then for each integer $r$ with $1 \leq r \leq q(X)$ there exists a $q(X)$-dimensional family $\left\{\mathscr{E}_{\alpha}\right\}_{\alpha \in \Gamma}$ of indecomposable aCM vector bundles of rank $r$ on $X$ such that $\mathscr{E}_{\alpha}$ for each $\alpha \in \Gamma$ is strictly semistable with $\operatorname{det}\left(\mathscr{E}_{\alpha}\right) \in$ $\operatorname{Pic}^{0}(X)$ and $c_{2}\left(\mathscr{E}_{\alpha}\right)=0$ with respect to any polarization of $X$, and there are only finitely many $\beta \in \Gamma$ with $\mathscr{E}_{\beta} \cong \mathscr{E}_{\alpha}$.

Proof. Fix a general line bundle $\mathscr{L} \in \operatorname{Pic}^{0}(X)$. Then as in Remark 4.2 we see that $\mathscr{L}$ is aCM. Set $\mathscr{G}_{0}=0$ the zero sheaf and $\mathscr{G}_{1}:=\mathscr{L}$. For an integer $r \geq 2$, we define $\mathscr{G}_{r}$ inductively as a general sheaf fitting into the following extension

$$
0 \rightarrow \mathscr{G}_{r-1} \stackrel{u}{\rightarrow} \mathscr{G}_{r} \stackrel{v}{\rightarrow} \mathscr{L} \rightarrow 0 .
$$

Note that $\mathscr{G}_{r}$ is strictly semistable for any polarization and $\mathscr{G}_{r} \otimes \mathscr{L}^{\vee}$ is an iterated extension of $\mathscr{O}_{X}$ for each $r \geq 1$. Since $\mathscr{G}_{r-1} \otimes \mathscr{L}^{\vee}$ is an iterated extension of $\mathscr{O}_{X}$, we have $\operatorname{det}\left(\mathscr{G}_{r-1} \otimes \mathscr{L}^{\vee}\right) \cong \mathscr{O}_{X}$ and $c_{2}\left(\mathscr{G}_{r-1} \otimes \mathscr{L}^{\vee}\right)=0$. Moreover, we may choose $\mathscr{G}_{r}$ admitting a non-trivial extension [10], because we have $\operatorname{ext}_{X}^{1}\left(\mathscr{L}, \mathscr{G}_{r-1}\right)>0$; indeed, we have $h^{1}\left(\mathscr{G}_{r-1} \otimes \mathscr{L}^{\vee}\right) \geq q(X)-r+2$, which is clearly true for $r=2$. In general, we get the following exact sequence from (10)

$$
H^{0}\left(\mathscr{O}_{X}\right) \rightarrow H^{1}\left(\mathscr{G}_{r-1} \otimes \mathscr{L}^{\vee}\right) \rightarrow H^{1}\left(\mathscr{G}_{r} \otimes \mathscr{L}^{\vee}\right) .
$$

Then we may apply the inductive hypothesis and $h^{0}\left(\mathscr{O}_{X}\right)=1$.

Note that the coboundary map $H^{0}\left(\mathscr{O}_{X}\right) \rightarrow H^{1}\left(\mathscr{G}_{r-1} \otimes \mathscr{L}^{\vee}\right)$ is zero if and only if (10) is the trivial extension. Since we take a non-trivial extension at each step, we have $h^{0}\left(\mathscr{G}_{r} \otimes \mathscr{L}^{\vee}\right)=h^{0}\left(\mathscr{G}_{r-1} \otimes \mathscr{L}^{\vee}\right)$. By induction on $r$ we get $h^{0}\left(\mathscr{G}_{r} \otimes \mathscr{L}^{\vee}\right)=1$ for all $r \leq q(X)$. Assume now that $\mathscr{G}_{r}$ is decomposable, say $\mathscr{G}_{r} \cong \mathscr{F}_{1} \oplus \mathscr{F}_{2}$ with each $\mathscr{F}_{i}$ nonzero. Then each $\mathscr{F}_{i} \otimes \mathscr{L}^{\vee}$ is a strictly semistable vector bundle with numerically trivial determinant. Since $\operatorname{gr}\left(\mathscr{G}_{r-1} \otimes \mathscr{L}^{\vee}\right)=\mathscr{O}_{X}^{\oplus(r-1)}$, we that $\operatorname{gr}\left(\mathscr{F}_{i} \otimes \mathscr{L}^{\vee}\right)$ is trivial and so each $\mathscr{F}_{i} \otimes \mathscr{L}^{\vee}$ has a subsheaf isomorphic to $\mathscr{O}_{X}$. In particular, we have $h^{0}\left(\mathscr{G}_{r} \otimes \mathscr{L}^{\vee}\right) \geq 2$, a contradiction.

Note that $\operatorname{det}\left(\mathscr{G}_{r}\right) \cong \mathscr{L}^{\otimes r}$ and so there are only finitely many line bundles $\mathscr{L}^{\prime} \in \operatorname{Pic}^{0}(X)$ such that $\mathscr{G}_{r}$ is also an iterated extension of $\mathscr{L}^{\prime}$. Hence we get the assertion from $\operatorname{dim} \operatorname{Pic}^{0}(X)=q(X)$.

Remark 4.4. Let $Y$ be a hyperelliptic surface, i.e. a smooth projective surface with $\omega_{Y} \nsucceq \mathscr{O}_{Y}, q(Y)=1$ and $\omega_{Y}^{\otimes 12} \cong \mathscr{O}_{Y}$. In particular, we have $h^{2}\left(\mathscr{O}_{Y}\right)=h^{0}\left(\omega_{Y}\right)=0$ and so $\chi\left(\mathscr{O}_{Y}\right)=0$. Let $X$ be a smooth projective surface birational to $Y$. Then we have $h^{i}\left(\mathscr{O}_{X}\right)=h^{i}\left(\mathscr{O}_{Y}\right)$ for each $i$ and $\omega_{X} \not \mathscr{O}_{X}$ with $h^{0}\left(\omega_{X}^{\otimes 12}\right)=1$. Fix an ample line bundle $\mathscr{O}_{X}(1)$ on $X$ and take a line bundle $\mathscr{L} \in \operatorname{Pic}^{0}(X) \backslash\left\{\mathscr{O}_{X}, \omega_{X}^{\vee}\right\}$. Then we have $h^{0}(\mathscr{L})=$ $h^{2}(\mathscr{L})=0$. Since $\mathscr{L}$ is numerically equivalent to $\mathscr{O}_{X}$ and $\chi\left(\mathscr{O}_{X}\right)=0$, we have $\chi(\mathscr{L})=0$ and so $h^{1}(\mathscr{L})=0$. Note that $\mathscr{L}(t)$ and $\mathscr{L}^{\vee} \otimes \omega_{X}(t)$ are ample for $t>0$, because they are numerically equivalent to the ample line bundle $\mathscr{O}_{X}(t)$. So we get $h^{1}(\mathscr{L}(t))=0$ for all $t \neq 0$ by Kodaira's vanishing and Serre's duality. Thus $\mathscr{L}$ is aCM. Now we may construct indecomposable aCM vector bundles $\mathscr{G}_{r}$ of rank $r$ as in the case of abelian surfaces. Indeed, we have $\operatorname{ext}_{X}^{1}(\mathscr{L}, \mathscr{L})=h^{1}\left(\mathscr{O}_{X}\right)=1$ and $\operatorname{ext}_{X}^{1}\left(\mathscr{L}, \mathscr{G}_{r-1}\right)>0$. We have $\operatorname{det}\left(\mathscr{G}_{r}\right) \cong \mathscr{L}^{\otimes r}$. In particular, there are only finitely many line bundles $\mathscr{L}^{\prime} \in \operatorname{Pic}^{0}(X)$ such that $\mathscr{G}_{r}$ is an iterated extension of $\mathscr{L}^{\prime}$. We get the following result from $q(X)=1$. 
Proposition 4.5. Let $X$ be a smooth projective surface, birational to a hyperelliptic surface, with any polarization. For any positive integer $r$, there exists a one-dimensional family $\left\{\mathscr{E}_{\alpha}\right\}_{\alpha \in \Gamma}$ of indecomposable aCM vector bundles of rank $r$ on $X$ such that for each $\alpha \in \Gamma$ there are only finitely many $\beta \in \Gamma$ with $\mathscr{E}_{\beta} \cong \mathscr{E}_{\alpha}$.

\section{SuRFACES OF GENERAL TYPE WITH AMPLE CANONICAL LINE BUNDLE}

Let $X$ be an integral projective surface, possibly singular, with ample $\omega_{X}$ satisfying the following conditions:

(i) $h^{1}\left(\omega_{X}^{\otimes n}\right)=0$ for all $n \in \mathbb{Z}$;

(ii- $\varepsilon) p_{g}:=h^{0}\left(\omega_{X}\right) \geq 2+\varepsilon$ with $\varepsilon \in\{0,1\}$.

We set $\mathscr{O}_{X}(1):=\omega_{X}$ with respect to which we consider aCM vector bundles on $X$.

Remark 5.1. Assume that $X$ is smooth. The canonical line bundle $\omega_{X}$ is ample if and only if $X$ is a minimal surface of general type without (-2)-curves, i.e. a smooth surface of general type without smooth rational curves $D \subset X$ with either $D^{2}=-1$ or $D^{2}=-2$; see [2]. There are surfaces $X$ of general type with $p_{g}=h^{0}\left(\omega_{X}\right) \leq 1$, but most surfaces have $p_{g} \geq 2$. The condition (i) for $n=0$ is $h^{1}\left(\mathscr{O}_{X}\right)=0$, i.e. the irregularity of $X$ is $q(X)=0$. This is a non-trivial requirement, but it is satisfied in many important cases. By Serre's duality this would imply that $h^{1}\left(\omega_{X}\right)=q(X)=0$. In characteristic 0 the condition (i) for $n<0$ comes from Kodaira's vanishing theorem by the ampleness of $\omega_{X}$. Assume $h^{1}\left(\omega_{X}^{\otimes n}\right)=0$ for all $n<0$. By Serre's duality we have $h^{1}\left(\omega_{X}^{\otimes n}\right)=h^{1}\left(\omega_{X}^{\otimes(1-n)}\right)=0$ for $n \geq 2$. Thus in characteristic 0 we have the condition (i) satisfied if and only if $h^{1}\left(\mathscr{O}_{X}\right)=0$.

By the condition (ii- $\varepsilon$ ), the set

$$
\Sigma:=\operatorname{Sing}(X) \cap\left(\text { the base locus of }\left|\omega_{X}\right|\right)
$$

is a proper closed subset of $X$. By the same argument in Remark 2.14 using Serre's duality we get the following lemma.

Lemma 5.2. For a finite subset $S \subset X \backslash \Sigma$, we have $\operatorname{ext}_{X}^{1}\left(\mathscr{I}_{S, X}, \omega_{X}\right)=|S|-1$ and a general extension of $\mathscr{I}_{S, X}$ by $\omega_{X}$ is locally free.

Proof. For the first assertion, we may apply the same argument in Remark2.14 using Serre's duality. The second assertion is clear, because the Cayley-Bacharach condition for $S$ and the linear system $\left|\mathscr{O}_{X}\right|$ is satisfied.

Proposition 5.3. For a fixed integer $2 \leq r \leq p_{g}$ and a general subset $S \subset X \backslash \Sigma$ with $|S|=r$, the general sheaf $\mathscr{E}$ fitting into an exact sequence

$$
0 \rightarrow \omega_{X}^{\oplus(r-1)} \rightarrow \mathscr{E} \rightarrow \mathscr{I}_{S, X} \rightarrow 0
$$

is an indecomposable and aCM vector bundle of rank $r$.

Proof. We get that $\mathscr{E}$ is locally free with rank $r$ from Lemma[5.2, Indeed, if $r \geq 3$, then $\mathscr{E} \cong \mathscr{G} \oplus \omega_{X}^{\oplus(r-2)}$ with a general extension $\mathscr{G}$ of $\mathscr{I}_{S, X}$ by $\omega_{X}$ is locally free. Then we may use openness of being locally free. Now since we have $\operatorname{ext}_{X}^{1}\left(\mathscr{I}_{S, X}, \omega_{X}\right)=r-1$ by Lemma 5.2 the extension (11) is induced by a choice of a basis $\left\{e_{1}, \ldots, e_{r-1}\right\}$ of $\operatorname{Ext}_{X}^{1}\left(\mathscr{I}_{S, X}, \omega_{X}\right)$. Thus the map $\varphi: H^{1}\left(\mathscr{I}_{S, X}\right) \rightarrow H^{2}\left(\omega_{X}^{\oplus(r-1)}\right) \cong \mathbf{k}^{\oplus(r-1)}$ is bijective, and in particular we have $h^{1}(\mathscr{E})=0$. Recall that we assume $\omega_{X} \cong \mathscr{O}_{X}(1)$. Then by the condition (i) we get $h^{1}\left(\omega_{X}(n)\right)=0$ for all $n \in \mathbb{Z}$ and we get

$$
0 \rightarrow H^{1}(\mathscr{E}(n)) \rightarrow H^{1}\left(\mathscr{I}_{S, X}(n)\right) \rightarrow H^{2}\left(\omega_{X}(n)\right)^{\oplus(r-1)} .
$$

Assume first that $n$ is positive and this implies $h^{2}\left(\omega_{X}(n)\right)=h^{0}\left(\mathscr{O}_{X}(-n)\right)=0$. Since $S$ is general with $|S|=r \leq h^{0}\left(\mathscr{O}_{X}(1)\right) \leq h^{0}\left(\mathscr{O}_{X}(n)\right)$, we get $h^{1}\left(\mathscr{I}_{S, X}(n)\right)=0$. Thus we have $h^{1}(\mathscr{E}(n))=0$. It remains to show that $h^{1}(\mathscr{E}(-n))=0$ for $n \geq 1$. In fact, it is sufficient to prove the existence of an extension $\mathscr{F}$ of $\mathscr{I}_{S, X}$ by $\omega_{X}^{\oplus(r-1)}$ satisfying $h^{1}(\mathscr{F}(-n))=0$ for all $n \geq 1$. Take $\mathscr{F} \cong \mathscr{G} \oplus \omega_{X}^{\oplus(r-2)}$ with a general extension $\mathscr{G}$ of $\mathscr{I}_{S, X}$ by $\omega_{X}$ given by $e_{1}$. By the previous argument, we have $h^{1}(\mathscr{G}(n))=0$ for all $n \geq 1$. By Lemma 5.2 $\mathscr{G}$ is locally 
free with $\operatorname{det}(\mathscr{G}) \cong \omega_{X}$. Serre's duality gives $h^{1}(\mathscr{G}(-n))=h^{1}(\mathscr{G}(n))=0$ for all $n \geq 1$. Thus we get that $\mathscr{E}$ is aCM. Note that if $r \geq 3$, then $\mathscr{G}$ is not aCM since we have $h^{1}(\mathscr{G})=r-2$.

For the indecomposability, we may use the same argument in the proof of Proposition 2.1 to $\mathscr{E} \otimes \omega_{X}^{\vee}$, because $\mathscr{I}_{S, X} \otimes \omega_{X}^{\vee}$ is indecomposable.

Now for the statement in Theorem[5.4, set $\varepsilon=r-2\left\lfloor\frac{r}{2}\right\rfloor$ for which the condition (ii- $\varepsilon$ ) for $X$ is assumed to be satisfied.

Theorem 5.4. For each integer $r \geq 2$, there exists an $r$-dimensional family $\left\{\mathscr{E}_{\alpha}\right\}_{\alpha \in \Gamma}$ of indecomposable aCM vector bundles of rank $r$ on $X$ with $\operatorname{det}\left(\mathscr{E}_{\alpha}\right) \cong \omega_{X}^{\otimes\lceil r / 2\rceil}$ and $c_{2}\left(\mathscr{E}_{\alpha}\right)=r$ such that for each $\alpha \in \Gamma$ there are only finitely many $\beta \in \Gamma$ with $\mathscr{E}_{\beta} \cong \mathscr{E}_{\alpha}$.

Proof. We use the same notations in the proof of Theorem 2.4 such as $\llbracket\left(S_{1}, \ldots, S_{i}\right)$ and $\mathbb{J}\left(S_{1}, \ldots, S_{i} ; S_{0}\right)$. Then we get the same assertions from Lemma2.5till Remark2.13, the only difference occurs in Lemma 2.8 and Remark2.9. where we have

$$
\operatorname{ext}_{X}^{1}\left(\mathscr{I}_{S_{i+1}, X}, \mathscr{J}\right)=\operatorname{ext}_{X}^{1}\left(\mathscr{I}_{S_{0}, X}, \mathscr{J}\right)=i
$$

for $\mathscr{J} \in \llbracket\left(S_{1}, \ldots, S_{i}\right)$ from $\operatorname{ext}_{X}^{1}\left(\mathscr{I}_{S_{i+1}, X}, \mathscr{O}_{X}\right)=\operatorname{ext}_{X}^{1}\left(\mathscr{I}_{S_{0}, X}, \mathscr{O}_{X}\right)=0$. Then we may consider the exact sequences (6) and (7) with $\mathscr{O}_{X}$ replaced by $\omega_{X}$.

\section{Surfaces mapped to a CURVE of Genus $\geq 3$ NOT AS Their Albanese imaGe}

Throughout this section, $X$ is a smooth projective surface admitting a surjective map $v: X \rightarrow C$ with $g=g(C) \geq 3$. Assume that $C$ is such a curve achieving maximum possible genus $g$ and that $q(X)>g$. For example, we may take as $X$ any smooth surface birational to $C \times D$, where $D$ is a smooth curve with $1 \leq g(D) \leq g$; in this case we have $q(X)=g+g(D)$.

Proposition 6.1. For each positive integer $r$ there exists a family $\left\{\mathscr{E}_{\alpha}\right\}_{\alpha \in \Gamma}$ of indecomposable aCM vector bundles of rank $r$ on $X$ such that $\Gamma$ is an integral variety with

$$
\operatorname{dim} \Gamma \geq q(X)+\frac{(r-1)(r-2)(g-1)}{2}-\frac{r(r-1)}{2}
$$

and each $\mathscr{E}_{\alpha}$ is strictly semistable with $\operatorname{det}\left(\mathscr{E}_{\alpha}\right) \in \operatorname{Pic}^{0}(X)$ and $c_{2}\left(\mathscr{E}_{\alpha}\right)=0$ with respect to any polarization of $X$ such that there are only finitely many $\beta \in \Gamma$ with $\mathscr{E}_{\beta} \cong \mathscr{E}_{\alpha}$.

Set $\mathscr{A}_{1}:=\mathscr{O}_{C}$ and define inductively a vector bundle $\mathscr{A}_{i+1}$ of rank $i+1$ on $C$ to be the middle term of the following extension:

$$
0 \rightarrow \mathscr{A}_{i} \rightarrow \mathscr{A}_{i+1} \rightarrow \mathscr{O}_{C} \rightarrow 0,
$$

where $\mathscr{A}_{i+1}=\mathscr{A}_{i+1}(e)$ corresponds to the extension class $e \in \operatorname{Ext}_{C}^{1}\left(\mathscr{O}_{C}, \mathscr{A}_{i}\right) \cong H^{1}\left(\mathscr{A}_{i}\right)$. The long exact sequence of cohomology of (12) gives $g-1 \leq h^{1}\left(\mathscr{A}_{i+1}\right)-h^{1}\left(\mathscr{A}_{i}\right) \leq g$. Since we have $g \geq 3$ from the assumption, we get $h^{1}\left(\mathscr{A}_{i+1}\right) \neq 0$. In particular, we may assume that the extension (12) is non-trivial. The image of the coboundary map $H^{0}\left(\mathscr{O}_{C}\right) \rightarrow H^{1}\left(\mathscr{A}_{i}\right)$ corresponds to the extension (12), up to a sign, and so we get $h^{0}\left(\mathscr{A}_{i+1}\right)=h^{0}\left(\mathscr{A}_{i}\right)$ and $h^{1}\left(\mathscr{A}_{i+1}\right)=h^{1}\left(\mathscr{A}_{i}\right)+g-1$ for each $i$. By induction, we get

$$
h^{0}\left(\mathscr{A}_{i}\right)=1 \text { and } h^{1}\left(\mathscr{A}_{i}\right)=i(g-1)+1 .
$$

Note that each $\mathscr{A}_{i}$ is an iterated extension of $\mathscr{O}_{C}$, and in particular it is strictly semistable with $\operatorname{gr}\left(\mathscr{A}_{i}\right) \cong$ $\mathscr{O}_{C}^{\oplus i}$. Assume $\mathscr{A}_{i} \cong \mathscr{B}_{1} \oplus \mathscr{B}_{2}$ with each $\mathscr{B}_{i} \neq 0$. Since each $\mathscr{B}_{i}$ has a HN-filtration with $\mathscr{O}_{C}$ as its first step, we have $h^{0}\left(\mathscr{B}_{i}\right)>0$ and so $h^{0}\left(\mathscr{A}_{i}\right) \geq 2$, a contradiction. Thus each $\mathscr{A}_{i}$ is indecomposable.

Remark 6.2. Let $u: \mathscr{A} \rightarrow \mathscr{B}$ be a surjection of sheaves on $C$. Since $\operatorname{dim} C=1$, we have $h^{2}(C, \operatorname{ker}(u))=0$. Thus the surjection $u$ induces a surjective map $H^{1}(C, \mathscr{A}) \rightarrow H^{1}(C, \mathscr{B})$. 
Lemma 6.3. Let $\mathscr{M}, \mathscr{D}_{1}, \mathscr{D}_{2}$ be vector bundles on $C$ fitting into exact sequences

$$
0 \rightarrow \mathscr{M} \stackrel{u_{i}}{\rightarrow} \mathscr{D}_{i} \rightarrow \mathscr{O}_{C} \rightarrow 0
$$

corresponding to an extension class $e_{i} \in \operatorname{Ext}_{C}^{1}\left(\mathscr{O}_{C}, \mathscr{M}\right) \cong H^{1}(\mathscr{M})$ for each $i$. If there exists an isomorphism $h: \mathscr{D}_{2} \rightarrow \mathscr{D}_{1}$ such that $h\left(u_{2}(\mathscr{M})\right)=u_{1}(\mathscr{M})$, then $e_{1}$ and $e_{2}$ are in the same orbit of $H^{1}(\mathscr{M})$ for the action of the group $\operatorname{Aut}(\mathscr{M})$.

Proof. Note that $h^{0}(\mathscr{M}) \leq h^{0}\left(\mathscr{D}_{i}\right) \leq h^{0}(\mathscr{M})+1$, and $h^{0}(\mathscr{M})=h^{0}\left(\mathscr{D}_{i}\right)$ if and only if $e_{i} \neq 0$. Since $h$ is an isomorphism, $e_{1}=0$ if and only if $e_{2}=0$. Since the assertion is obvious when $e_{1}=e_{2}=0$, we may assume $e_{1} \neq 0$ and $e_{2} \neq 0$. Since $h\left(u_{2}(\mathscr{M})\right)=u_{1}(\mathscr{M}), h$ induces isomorphisms $h^{\prime}: D_{2} / u_{2}(\mathscr{M}) \rightarrow \mathscr{D}_{1} / u_{1}(\mathscr{M})$ and $f: \mathscr{M} \rightarrow \mathcal{M}$. Since $\mathscr{D}_{i} / u_{i}(\mathscr{M}) \cong \mathscr{O}_{C}, i=1,2, h^{\prime}$ is induced by the multiplication by a constant, $c$. Note that $e_{i}$ is determined by the image of 1 by the coboundary map $H^{0}\left(\mathscr{O}_{C}\right) \rightarrow H^{1}(\mathscr{M})$ in (13). Since $e_{1} \neq 0$ and $e_{2} \neq 0$, we have $c \neq 0$. Taking $\left(\frac{1}{c}\right) h$ instead of $h$ we reduce to the case in which $h^{\prime}: \mathscr{O}_{C} \rightarrow \mathscr{O}_{C}$ is the identity map. Thus we get a commutative diagram with exact rows:

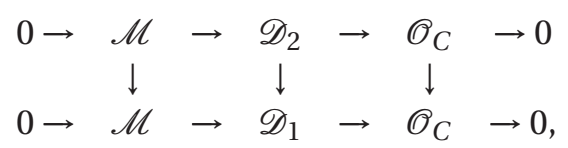

in which the three vertical arrows are respectively $f, h$ and $\operatorname{Id}_{\mathscr{O}_{C}}$. By the definition of $\operatorname{Ext}_{C}^{1}\left(\mathscr{O}_{C}, \mathscr{M}\right)$ as short exact sequences modulo an equivalence relation, we get $e_{1}=f_{*}\left(e_{2}\right)$, i.e. $e_{1} \in H^{1}(\mathscr{M})$ is contained in the orbit of $e_{2}$ for the action of the group $\operatorname{Aut}(\mathscr{M})$.

We set $\mathbf{T}_{2}:=H^{1}\left(\mathscr{O}_{C}\right) \backslash\{0\}$ and consider it as a parameter space, not finite-to-one, for non-trivial extensions of $\mathscr{O}_{C}$ by $\mathscr{O}_{C}$. Then we get a family $\left\{\mathscr{A}_{2}(e)\right\}_{e \in \mathbf{T}_{2}}$ of aCM vector bundles of rank two. Since we have $h^{1}\left(\mathscr{A}_{2}(e)\right)=2 g-3$ for each $e \in \mathbf{T}_{2}$, there is a vector bundle $\pi_{2}: \mathbf{T}_{3}^{\prime} \rightarrow \mathbf{T}_{2}$ of rank $2 g-3$ whose fibre over $\mathscr{A}_{2}(e)$ is $H^{1}\left(\mathscr{A}_{2}(e)\right) \cong \operatorname{Ext}_{C}^{1}\left(\mathscr{O}_{C}, \mathscr{A}_{2}(e)\right)$. Then we get a family $\left\{\mathscr{A}_{3}(e)\right\}_{e \in \mathbf{T}_{3}^{\prime}}$ of aCM vector bundles of rank three on $C$ such that for each $e \in \mathbf{T}_{2}^{\prime}, \mathscr{A}_{3}(e)$ is an extension of $\mathscr{O}_{C}$ by $\mathscr{A}_{2}(\pi(e))$. Let $\mathbf{T}_{3}$ be the non-empty Zariski open subset of $\mathbf{T}_{3}^{\prime}$ parametrizing the non-trivial extensions of $\mathscr{O}_{C}$ by $\mathscr{A}_{2}(\pi(e))$. Thus we have a family $\left\{\mathscr{A}_{3}(e)\right\}_{e \in \mathbf{T}_{3}}$ of indecomposable aCM vector bundles of rank three, parametrized by $\mathbf{T}_{3}$.

Now we define a parameter space $\mathbf{T}_{i}$ inductively: fix an integer $i \geq 2$ and assume that $\mathbf{T}_{i}$ is defined, together with a family $\left\{\mathscr{A}_{i}(e)\right\}_{e \in \mathbf{T}_{i}}$ of indecomposable aCM vector bundles of rank $i$, parametrized by $\mathbf{T}_{i}$. Since we have $h^{1}\left(\mathscr{A}_{i}(e)\right)=i(g-1)+1$, there exists a vector bundle $\pi_{i}: \mathbf{T}_{i+1}^{\prime} \rightarrow \mathbf{T}_{i}$ of rank $i(g-1)+1$ and a family $\left\{\mathscr{A}_{i+1}(e)\right\}_{e \in \mathbf{T}_{i+1}^{\prime}}$ of aCM vector bundles of rank $i+1$ on $C$ such that for each $e \in \mathbf{T}_{i+1}^{\prime}, \mathscr{A}_{i+1}(e)$ is an extension of $\mathscr{O}_{C}$ by $\mathscr{A}_{i}(\pi(e))$. Let $\mathbf{T}_{i+1}$ be the non-empty Zariski open subset of $\mathbf{T}_{i+1}^{\prime}$ parametrizing the non-trivial extensions of $\mathscr{O}_{C}$ by $\mathscr{A}_{i}(\pi(e))$.

If a vector bundle $\mathscr{A}=\mathscr{A}_{r}$ of rank $r$ on $C$ corresponding to $e \in \mathbf{T}_{r}$ is obtained as a successive extension of $\mathscr{O}_{C}$ by $\mathscr{A}_{i}\left(e_{i-1}\right)$ corresponding to $e_{i} \in H^{1}\left(\mathscr{A}_{i}\left(e_{i-1}\right)\right) \backslash\{0\}$ for each $i \leq r$, then we simply denote it by $\mathscr{A}\left(e_{1}, \ldots, e_{r-1}\right):=\mathscr{A}$ and it has a filtration

$$
0 \subset \mathscr{A}_{1}=\mathscr{O}_{C} \subset \mathscr{A}_{2}=\mathscr{A}\left(e_{1}\right) \subset \mathscr{A}_{3}=\mathscr{A}\left(e_{1}, e_{2}\right) \subset \cdots \subset \mathscr{A}_{r}=\mathscr{A}\left(e_{1}, \ldots, e_{r-1}\right) .
$$

Fix a general $\mathscr{A}=\mathscr{A}\left(e_{1}, \ldots, e_{r-1}\right)$ that is a non-trivial extension of $\mathscr{O}_{C}$ by $\mathscr{A}^{\prime}:=\mathscr{A}\left(e_{1}, \ldots, e_{r-2}\right)$. Letting $u_{i, r}: \mathscr{A}_{i} \rightarrow \mathscr{A}$ with $1 \leq i \leq r-1$ be the inclusion arising by the extensions reaching $\mathscr{A}$, we have the 
following commutative diagram

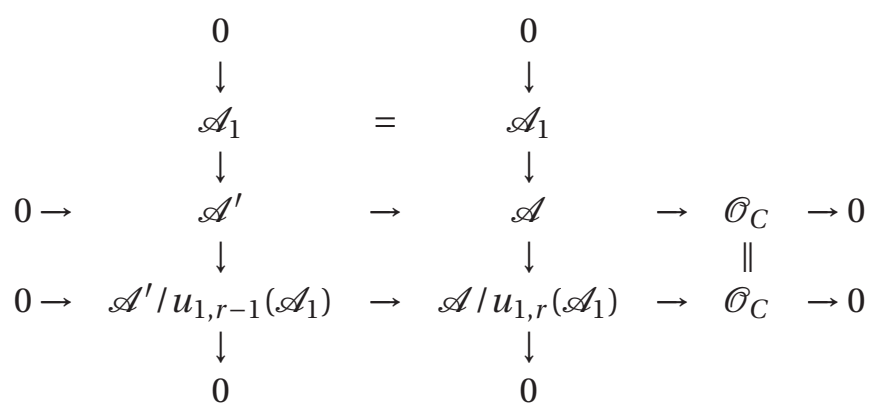

so that $\mathscr{A} / u_{1, r}\left(\mathscr{A}_{1}\right)$ is an extension of $\mathscr{O}_{C}$ by $\mathscr{A}^{\prime} / u_{1, r}\left(\mathscr{A}_{1}\right)$. Iterating the process, we see that $\mathscr{A} / u_{1, r}\left(\mathscr{A}_{1}\right)$ is an iterated extension of $\mathscr{O}_{C}$.

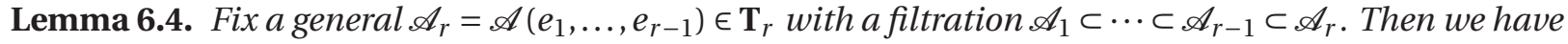

(i) $h^{0}\left(\mathscr{A}_{i} / \mathscr{A}_{j}\right)=1$ for all $1 \leq j<i \leq r$;

(ii) $f\left(\mathscr{A}_{i}\right) \subset \mathscr{A}_{i}$ for any $f \in \operatorname{End}\left(\mathscr{A}_{r}\right)$ and each $i$;

(iii) $\operatorname{dim} \operatorname{End}\left(\mathscr{A}_{r}\right) \leq r$ and $\operatorname{dim} \operatorname{End}\left(\mathscr{A}_{r}\right)-\operatorname{dim}\left(\mathscr{A}_{r-1}\right) \leq 1$.

(iv) $h\left(\mathscr{A}_{i}\right)=\mathscr{B}_{i}$ for all $i$ and any isomorphism $h: \mathscr{B}_{r} \rightarrow \mathscr{A}_{r}$, where $\mathscr{B}_{r} \in \mathbf{T}_{r}$ general with a filtration $\mathscr{B}_{1} \subset \cdots \subset \mathscr{B}_{r-1} \subset \mathscr{B}_{r}$.

Proof. For (i) consider the following sequence, obtained from (12):

$$
0 \rightarrow \mathscr{A}_{i} / \mathscr{A}_{j} \rightarrow \mathscr{A}_{i+1} / \mathscr{A}_{j} \rightarrow \mathscr{O}_{C} \rightarrow 0 \text {. }
$$

Since $e_{i} \in H^{1}\left(\mathscr{A}_{i}\right)$ is general by the generality of $\mathscr{A}_{r}$, we get that (14) is a general extension and $h^{0}\left(\mathscr{A}_{i+1} / \mathscr{A}_{j}\right)=$ $h^{0}\left(\mathscr{A}_{i} / \mathscr{A}_{j}\right)$. Thus to prove the assertion for $j=1$ it is enough to show it for the case $i=2$, which is obvious from $\mathscr{A}_{2} / \mathscr{A}_{1} \cong \mathscr{O}_{C}$. For $j \geq 2$ we use (14) starting from the case $i=j+1$, when we have $\mathscr{A}_{j+1} / \mathscr{A}_{j} \cong \mathscr{O}_{C}$.

For (ii) note first that $\mathscr{A}_{1}=\mathscr{O}_{C}$ and $h^{0}\left(\mathscr{A}_{r}\right)=1$. This implies that $\mathscr{A}_{1}$ is the image of the evaluation map $H^{0}\left(\mathscr{A}_{r}\right) \otimes \mathscr{O}_{C} \rightarrow \mathscr{A}_{r}$ and so $f\left(\mathscr{A}_{1}\right) \subseteq \mathscr{A}_{1}$, concluding the case $r=2$. Now $f$ induces a map $f^{\prime}: \mathscr{A}_{r} / \mathscr{A}_{1} \rightarrow$ $\mathscr{A}_{r} / \mathscr{A}_{1}$. Since $h^{0}\left(\mathscr{A}_{r} / \mathscr{A}_{1}\right)=1$ by (i) and $\mathscr{A}_{2} / \mathscr{A}_{1} \cong \mathscr{O}_{C}$, we get $f^{\prime}\left(\mathscr{A}_{2} / \mathscr{A}_{1}\right) \subseteq \mathscr{A}_{2} / \mathscr{A}_{1}$ and so $f\left(\mathscr{A}_{2}\right) \subseteq \mathscr{A}_{2}$. Thus we get the assertion by continuing this process together with (i).

For (iii) since the case $r=1$ is trivial, we may assume $r \geq 2$ and use induction on $r$. For $f \in \operatorname{End}\left(\mathscr{A}_{r}\right)$, we have $\mathscr{A}_{1}=\mathscr{O}_{C}$ and $f\left(\mathscr{A}_{1}\right) \subseteq \mathscr{A}_{1}$ by (ii). Thus there is $c \in \mathbf{k}$ such that $\left(f-c \cdot \operatorname{Id}_{\mathscr{A}_{r}}\right)\left(\mathscr{A}_{1}\right)=0$, and $f-c \cdot \operatorname{Id}_{\mathscr{A}_{r}}$ is uniquely determined by $f^{\prime} \in \operatorname{End}\left(\mathscr{A}_{r} / \mathscr{A}_{1}\right)$. Since we may apply (i) and (ii) to $\mathscr{A}_{r} / \mathscr{A}_{1}$, we conclude by induction on $r$.

For (iv) note that $\mathscr{A}_{1}$ (resp. $\mathscr{B}_{1}$ ) is the image of the evaluation map of $\mathscr{A}_{r}$ (resp. $\mathscr{B}_{r}$ ) and $h$ is an isomorphism. In particular, we have $h\left(\mathscr{A}_{1}\right)=\mathscr{B}_{1}$ and so $h$ induces an isomorphism $h^{\prime}: \mathscr{A}_{r} / \mathscr{A}_{1} \rightarrow \mathscr{B}_{r} / \mathscr{B}_{1}$. Since $h^{0}\left(\mathscr{A}_{i} / \mathscr{A}_{j}\right)=h^{0}\left(\mathscr{B}_{i} / \mathscr{B}_{j}\right)=1$ for all $i>j$ by (i), we iterate the previous argument.

Define a subset $\mathbf{J}_{r}$ to be

$$
\mathbf{J}_{r}=\left\{\begin{array}{l|l}
e \in \mathbf{T}_{r} & \begin{array}{c}
\mathscr{A}_{r}(e) \text { admits a filtration } \mathscr{A}_{1} \subset \cdots \subset \mathscr{A}_{r-1} \subset \mathscr{A}_{r} \\
\text { such that } h^{0}\left(\mathscr{A}_{i} / \mathscr{A}_{j}\right)=1 \text { for all } 1 \leq j<i \leq r
\end{array}
\end{array}\right\},
$$

i.e. the non-empty open subset of $\mathbf{T}_{r}$ parametrizing the vector bundles $\mathscr{A}_{r}$ satisfying (i) of Lemma 6.4 thus $\mathscr{A}_{r}$ satisfies (ii), (iii) and (iv) of Lemma6.4

Lemma 6.5. For a general $\mathscr{A}_{r} \in \mathbf{J}_{r}$ there exists an algebraic subset of $\mathbf{J}_{r}$, parametrizing the vector bundles isomorphic to $\mathscr{A}_{r}$, with dimension at most $\frac{r(r-1)}{2}$.

Proof. We use induction on $r$; the case $r=1$ is trivial, because $\mathbf{J}_{1}=\mathbf{T}_{1}=\left\{\mathscr{O}_{C}\right\}$. We assume that $r \geq 2$ and fix $\mathscr{B}_{r} \in \mathbf{J}_{r}$, isomorphic to $\mathscr{A}_{r}$, with a filtration $\mathscr{B}_{1} \subset \cdots \subset \mathscr{B}_{r}$. For any isomorphism $h: \mathscr{B}_{r} \rightarrow \mathscr{A}_{r}$, we have $h\left(\mathscr{B}_{r-1}\right)=\mathscr{A}_{r-1}$ by (iv) of Lemma6.4. Since $\mathscr{A}_{r-1}$ is also general in $\mathbf{J}_{r-1}$, by inductive assumption there is an algebraic subset $\mathbf{J}^{\prime}$ of $\mathbf{J}_{r-1}$ parametrizing the vector bundles isomorphic to $\mathscr{A}_{r-1}$. Fix $\mathscr{M} \in \mathbf{J}^{\prime}$ and 
consider the subset $\mathbf{T}^{\prime} \subset \mathbf{T}_{r}$ of all extensions of $\mathscr{O}_{C}$ by $\mathscr{M}$ which are isomorphic to $\mathscr{A}_{r}$. By Lemma6.3 and (iii) of Lemma 6.4, we have $\operatorname{dim} \mathbf{T}^{\prime} \leq r-1$ and we get the assertion.

Proof of Proposition 6.1; Note that

$$
g-1+\sum_{i=2}^{r-1}(i(g-1)-1)-\sum_{i=1}^{r-1} i=\frac{(r-1)(r-2)(g-1)}{2}-\frac{r(r-1)}{2} .
$$

Set $\Delta:=\left\{v^{*}(\mathscr{A}) \mid \mathscr{A} \in \mathbf{J}_{r}\right\}$ and then each element of $\Delta$ is indecomposable, because each $\mathscr{A} \in \mathbf{J}_{r}$ is indecomposable. Since we have $v_{*} v^{*} \mathscr{F} \cong \mathscr{F}$ for any vector bundle $\mathscr{F}$ on $C$ by the projection formula and $v_{*} \mathscr{O}_{X} \cong \mathscr{O}_{C}$, we have $v^{*} \mathscr{A} \cong v^{*} \mathscr{B}$ if and only if $\mathscr{A} \cong \mathscr{B}$ for any $v^{*} \mathscr{A}, v^{*} \mathscr{B} \in \Delta$.

Fix a general $\mathscr{L} \in \operatorname{Pic}^{0}(X)$ and set $\Theta_{\mathscr{L}}:=\{\mathscr{G} \otimes \mathscr{L} \mid \mathscr{G} \in \Delta\}$. Each element of $\Theta_{\mathscr{L}}$ is an indecomposable vector bundle of rank $r$ on $X$ and the isomorphism classes of elements in $\Theta_{\mathscr{L}}$ are also parametrized by $\mathbf{J}_{r}$. We have $h^{1}(\mathscr{L})=0$ by [3, Th. 0.1], because $q(X)>g$ and by our definition of $g$ there is no nonconstant morphism from $X$ to a curve of genus $q(X)$. Fix a positive interger $t$. By Kleiman's numerical criterion of ampleness in [17], $\mathscr{L}^{\vee}(t)$ and $\omega_{X}^{\vee} \otimes \mathscr{L}(t)$ are ample. So Kodaira' vanishing gives $h^{1}(\mathscr{L}(-t))=$ $h^{1}\left(\omega_{X} \otimes \mathscr{L}^{\vee}(-t)\right)=0$. On the other hand, by Serre's duality we get $h^{1}(\mathscr{L}(t))=h^{1}\left(\omega_{X} \otimes \mathscr{L}^{\vee}(-t)\right)=0$. Thus $\mathscr{L}$ is aCM.

Since each element of $\Theta_{\mathscr{L}}$ is an iterated extension of $\mathscr{L}$, each element of $\Theta_{\mathscr{L}}$ is also aCM. Note that each element of $\Theta_{\mathscr{L}}$ is strictly semistable with $\operatorname{gr}\left(\mathscr{A}_{r}\right) \cong \mathscr{L}^{\oplus r}$ and so no element of $\Theta_{\mathscr{L}}$ is isomorphic to an element of $\Theta \mathscr{L}^{\prime}$ with $\mathscr{L} \not \mathscr{L}^{\prime}$. Now we may vary the general $\mathscr{L} \in \operatorname{Pic}^{0}(X)$ to obtain a family $\Gamma$ whose fibre over $\mathscr{L}$ is $\Theta \mathscr{L}$. Then we get the inequality in the assertion and all the requirements for $\Gamma$ are satisfied.

\section{REFERENCES}

1. M. F. Atiyah, Vector bundles over an elliptic curve. Proc. London Math. Soc. (3) 7 (1957), 414-452.

2. W. Barth, K. Hulek, Ch. Peters and A. Van de Ven, Compact complex surfaces, Erg. Math., 3. Folge, Springer Verlag, Berlin (2004).

3. A. Beauville, Annulation du $H^{1}$ et systemes paracaniques sur les surfaces, J. Reine Angew. Math. 388 (1988), $149-157$.

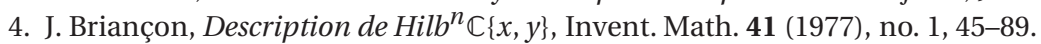

5. J. Briançon and A. Iarrobino, Dimension of the punctual Hilbert scheme, J. Algebra 55 (1978), no. 2, 536-544.

6. G. Casnati, Special Ulrich bundles on non-special surfaces with $p_{g}=q=0$, Internat. J. Math. 8 (2017), no. 8, 18p.

7. G. Casnati, Ulrich bundles on non-special surfaces with $p_{g}=0, q=1$, to appear in Rev. Mat. Complutense, arXiv:1707.06429.

8. D. Eisenbud and J. Herzog, The classification of homogeneous Cohen-Macaulay rings of finite representation type, Math. Ann. 280 (1988), no. 2, 347-352.

9. D. Faenzi and F. Malaspina, Surfaces of minimal degree of tame representation type and mutations of Cohen-Macaulay modules, Adv. Math. 310 (2017), 663-695.

10. D. Faenzi and J. Pons-Llopis, The Cohen-Macaulay representation type of arithmetically Cohen-Macaulay varieties, preprint, arXiv:1504.03819v2 [math.AG].

11. M. Granger, Géometrie des schémas de Hilbert ponctuels, Mem. Math. Soc. France 8, 1983.

12. M. Green and R. Lazarsfeld, Deformation theory, generic vanishing theorems, and some conjectures of Enriques, Catanese and Beauville, Invent. Math. 90 (1987), no. 2, 389-407.

13. M. Green and R. Lazarsfeld, Higher obstructions to deforming cohomology groups of line bundles, J. Amer. Math. Soc. 4 (1991), no. 1, 87-103.

14. R. Hartshorne, Algebraic geometry, Springer-Verlag, New York-Heidelberg, 1977, Graduate Texts in Mathematics, No. 52.

15. G. Horrocks, Vector bundles on the punctured spectrum of a local ring, Proc. London Math. Soc. 3 (1964) no. 14. 689-713.

16. A. Iarrobino, Punctual Hilbert scheme, Mem. Amer. Math. Soc. 188, 1977.

17. S. L. Kleiman, Toward a numerical theory of ampleness, Ann. Math. 84 (1966), 293-344.

18. S. L. Kleiman, and J. Landolfi, Geometry and deformation of special Schubert varieties, Compositio Math. 23 (1971), $407-434$.

19. H. Knörrer, Cohen-Macaulay modules on hypersurface singularities. I, Invent. Math. 88 (1987), no. 1, $153-164$.

20. H. Lange, Universal families of extensions, J. Algebra 83 (1983), 101-112.

21. S. Mukai, Semi-homogeneous vector bundles on an Abelian variety. J. Math. Kyoto Univ. 18 (1978), no. 2, $239-272$.

22. J. Pons-Llopis, Non-arithmetically Cohen Macaulay schemes of wild representation type, Manuscripta Mathematica (2018), https://doi.org/10.1007/s0022.

23. K. Ueno, Classification theory of algebraic varieties and compact complex spaces. Notes written in collaboration with P. Cherenack, Lecture Notes in Mathematics, Vol. 439. Springer-Verlag, Berlin-New York, 1975. xix+278 pp. 
24. C. A. Weibel, An introduction to homological algebra, Cambridge University Press, vol. 38, 1995.

Università Di Trento, 38123 Povo (TN), ITALY

E-mail address: edoardo.ballico@unitn.it

SUNGKYUNKWAN UNIVERSITY, SUWON 440-746, KoREA

E-mail address: sukmoonh@skku.edu

Department of Information EngineERIng, Computer SCIENCE AND MATHEMATiCs - UniVersity Of L'AQUiLA, ITALY E-mail address: juanfrancisco.ponsllopis@univaq.it 